\title{
Influence of a planar metal nanoparticle assembly on the optical response of a quantum emitter
}

\author{
Harini Hapuarachchi ${ }^{*}{ }^{*}$ and Jared H. Cole ${ }^{\dagger}{ }^{\dagger}$ \\ ARC Centre of Excellence in Exciton Science and Chemical and Quantum Physics, School of Science, \\ RMIT University, Melbourne, 3001, Australia
}

(Received 12 June 2020; revised 9 September 2020; accepted 15 September 2020; published 16 October 2020)

\begin{abstract}
We develop an analytical framework to study the influence of a weakly intercoupled in-plane spherical metal nanoparticle (MNP) assembly on a coherently illuminated quantum emitter (QE). We reduce the analytical expressions derived for the aforementioned generic planar setup into simple and concise expressions representing a QE mediated by a symmetric MNP constellation, by exploiting the symmetry. We use the recently introduced generalized nonlocal optical response (GNOR) theory that has successfully explained plasmonic experiments to model the MNPs in our system. Due to the use of GNOR theory, and our analytical approach, the procedure we suggest is extremely computationally efficient. Using the derived model, we analyze the absorption rate, resultant Rabi frequency, effective excitonic energy shift, and dephasing rate shift spectra of an exciton bearing QE at the center of a symmetric MNP setup. We observe that the QE experiences plasmon-induced absorption rate spectral linewidth variations that increase in magnitude with decreasing MNP-QE center separation and increasing number of MNPs. Our results also suggest that parameter regions where the QE exhibits trends of decreasing linewidth against decreasing MNP-QE center separation are likely to be associated with plasmon-induced excitonic energy redshifts. Similarly, regions where the QE absorption rate linewidth tends to increase against decreasing MNP-QE center separation are likely to be accompanied by plasmon-induced excitonic energy blueshifts. In both these cases, the magnitude of the observed redshift and blueshift was seen to increase with the number of MNPs in the constellation, due to enhancement of the plasmonic influence. We show that even when the magnitude of the QE absorption rate spectrum is much smaller compared to the isolated (collective) MNP spectra, it is sufficient to dramatically modify the spectrum of the MNP-QE nanohybrid, causing sharp Fano-type interference patterns.
\end{abstract}

DOI: 10.1103/PhysRevResearch.2.043092

\section{INTRODUCTION}

Metamaterials formed by combining different types of nanoparticles are gaining increasing research attention due to their unprecedented capabilities to manipulate light at the nanoscale [1,2]. Plasmonic metal nanoparticles (MNPs) [3] and quantum emitters (QEs) [4] are two categories of widely studied nanoparticles whose fascinating optoelectronic properties are often expected to synergize when combined [5-8]. Due to the tunability of the optical properties using their size and structure, MNPs and QEs possess a wide array of applications in a variety of fields such as biosensing $[9,10]$, photothermal cancer therapy $[11,12]$, optoelectronic nanodevices [13-18], and photovoltaics [19,20]. When a QE is kept in nanoscale proximity to a small MNP, a dipole-dipole coupling occurs between the two nanoparticles forming a highly tunable hybrid nanosystem exhibiting interesting optical signatures $[1,2,21]$. It has been shown theoretically, as well as experimentally, that hybrid superstructures where one or more

\footnotetext{
*harini.hapuarachchi@rmit.edu.au

†jared.cole@rmit.edu.au

Published by the American Physical Society under the terms of the Creative Commons Attribution 4.0 International license. Further distribution of this work must maintain attribution to the author(s) and the published article's title, journal citation, and DOI.
}

MNPs are attached to QEs have the potential to be utilized as versatile sensors and actuators which can surpass the capabilities of the individual constituents [11,22]. Therefore, hybrid molecules made of MNPs and QEs have captured the attention of both theorists and experimentalists. Moreover, various techniques to successfully fabricate MNP-QE nanohybrids that can be probed at the single-molecule level have already been demonstrated in the literature [23-25]. In this context, attempts to enhance our understanding of systems comprising multiple MNPs and QEs are of vital importance [26,27].

MNPs much smaller than the wavelength of the incident light $(\lambda)$ exhibit strong dipolar resonant excitations known as localized surface plasmon resonances (LSPRs) [28,29]. These resonances enable MNPs to act as nanoscale optical cavities that are able to focus electromagnetic energy to spots much smaller than $\lambda$, overcoming the half-wavelength size limitation of the conventional optical cavities [30,31]. LSPRs are nonpropagating modes of excitation of the conduction band electrons which arise naturally from the scattering problem of a subwavelength MNP in an oscillating electromagnetic field. An effective restoring force is exerted on the driven electrons by the curved surface of the particle, which leads to an amplification of the field, both inside the particle, and on the near field of the outside. The resonant condition of this phenomenon is termed a localized surface plasmon resonance [3]. In frequency regions close to their plasmonic peaks, MNPs can be used to tailor the optical response of nearly resonant QEs [11,32,33]. 
It is evident that the optical response of each participating MNP plays a pivotal role in determining the behavior of the MNP-QE hybrid nanostructures. The most widely adopted analytical approach in the literature to model the optical response of plasmonic nanoparticles such as MNPs is the use of classical local response approximation (LRA) [22,3335]. However, this approach overlooks the nonlocal effects $[34,36]$ that become prominent in small MNPs, where the ratio of the number of surface atoms to those that make up the bulk of the particle is significant [2]. Thus, LRA has been challenged on a number of accounts, for example, its prediction that the surface plasmon resonance energy in the quasistatic limit is independent of the MNP size, which conflicts with the experimentally observed results $[34,37-$ 40]. Such nonclassical effects could be captured using $a b$ initio approaches such as density-functional theory (DFT) [41,42]. However, such approaches are extremely computationally demanding, especially for nanohybrids formed by coupling several particles together. A simpler and computationally less demanding approach would be to surpass the LRA using nonlocal response theories such as the nonlocal hydrodynamic model or the generalized nonlocal optical response (GNOR) theory [34]. The GNOR theory is a recent generalization and an extension of the nonlocal hydrodynamic model, which goes beyond the latter by taking both convection current and electron diffusion phenomena in the MNPs into account $[34,38]$. It has been shown to better capture both size-dependent resonance shifts and linewidth broadening of the MNP extinction cross section that occurs with decreasing particle size. It has been shown that GNOR based absorption spectra of bare Ag nanoparticles closely align with the respective experimental electron energy-loss spectroscopy (EELS) observations compared to theoretical predictions obtained using tools such as discrete dipole approximation (DDA) and coordinate-dependent discrete interaction model (cd-DIM) which is an atomistic variant of DDA [43]. GNOR also successfully approximates experimentally measured spectra for both monomers and dimers (with nanometer-sized gaps) which previously seemed to require microscopic theory and invocation of the quantum mechanical effects of tunneling $[34,36]$.

In this paper, we develop a comprehensive analytical framework to study the interaction of a coherently illuminated two-level quantum emitter with a nonlocally modeled in-plane assembly of spherical MNPs. We utilize the GNOR theory $[34,36]$ to model the MNPs in our assembly. The resulting procedure is extremely computationally efficient.

This paper is organized as follows: In Sec. II A, we first outline the theoretical foundations of modeling MNP dipoles using the GNOR theory, followed by Secs. IIB and IIC, where we analytically derive the complete forms of the external-field-induced and QE-induced dipoles in the generic planar MNP constellation. In Sec. II D, we derive the effective external field experienced by the coherently illuminated QE, under the influence of the in-plane MNP dipole assembly. In Sec. IIE, we reduce the equations derived in the earlier sections to obtain simplified, elegant analytical equations for a planar symmetric setup. Then, we model the $\mathrm{QE}$ as an open quantum system in Sec. IIF, and outline the procedure of obtaining the steady-state solutions to the QE density matrix

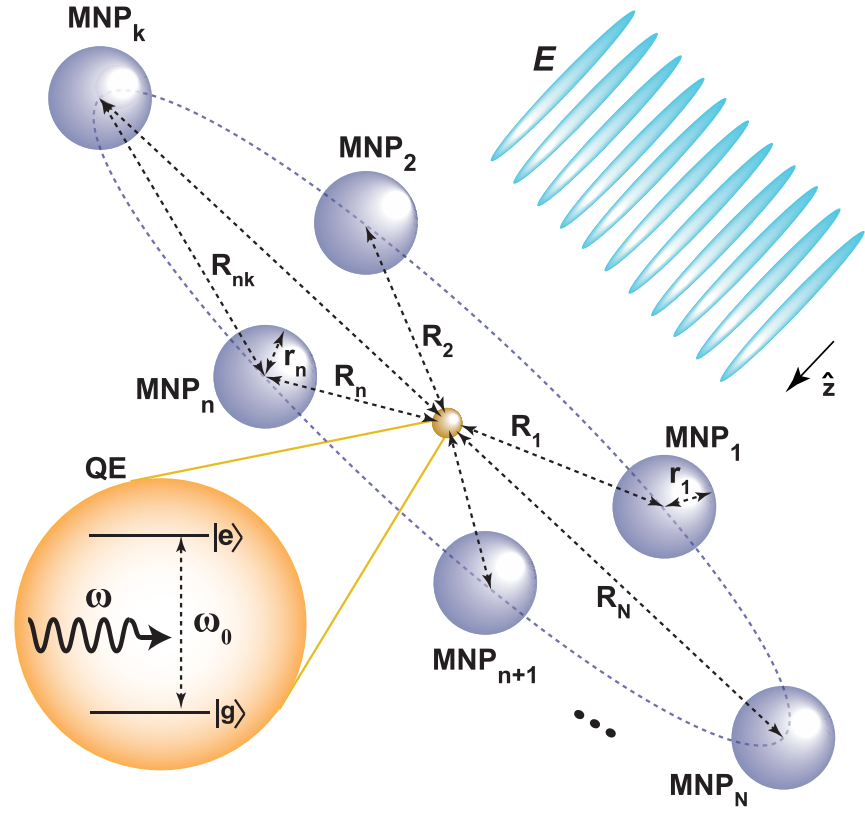

FIG. 1. Schematic diagram of the system under study. The exciton bearing quantum emitter undergoes dipole interactions with the coherent external drive $\boldsymbol{E}$ (incident along the $z$ axis) and the dipole response fields of the metal nanoparticles placed around it, in the $x y$ plane.

in Sec. II G. Finally, in Sec. III, we use the derived model for a detailed analysis of a QE under the influence of a weakly intercoupled in-plane symmetric MNP setup.

\section{FORMALISM}

We consider $N$ spherical MNPs of radius $r_{\mathrm{n}}$, placed at center-to-center separations $R_{\mathrm{n}}$ from a $\mathrm{QE}$, where $\mathrm{n}=$ $1, \ldots, N$. This setup, depicted in Fig. 1, is lying in the $x y$ plane. The exciton bearing quantum emitter (QE) is assumed to have a relatively negligible radius, a relative permittivity $\epsilon_{\mathrm{s}}$, and an excitonic energy $\hbar \omega_{0}$. A perpendicularly incident coherent electric field $\boldsymbol{E}=E_{0}\left(e^{-i \omega t}+e^{i \omega t}\right) \hat{z}=E \hat{z}$ optically couples the components of our hybrid nanosystem, where $\omega$ is the angular frequency of the incoming radiation and $\hat{z}$ is the unit vector along the $z$ axis. The system is submerged in a bath of relative permittivity $\epsilon_{\mathrm{b}}$, and transient thermal effects $[12,44]$ are assumed to be negligible. We assume reasonably sufficient MNP separations to neglect inter-MNP quantum mechanical $[45,46]$ and multipolar hybridization effects $[26,47,48]$, under perpendicular polarization conditions.

Note that throughout the formalism section we use bold fonts, hat notation, tilde notation, plus sign superscript, and bold hat notation to refer to vectors, quantum mechanical operators, slowly varying amplitudes, positive-frequency components, and unit vectors, respectively.

\section{A. Nonlocal model for plasmonic dipoles}

For our planar MNP constellation, we consider small metallic spheres, each with radius $r_{\mathrm{n}} \ll \lambda$ (wavelength of incoming radiation). For such MNPs the ideal dipole representation is valid in the quasistatic regime, which allows 
for time-varying fields but neglects the effects of spatial retardation over the particle volume [3]. The system of metal nanoparticles is described by the coupled dipole approximation under the quasistatic limit, where one MNP's influence on the other can be simplified to that of a point dipole at the origin of the former MNP [26]. The dielectric permittivity of each MNP is obtained using the Drude-type dielectric function [34]

$$
\epsilon_{\mathrm{m}}(\omega)=\epsilon_{\mathrm{core}}(\omega)-\frac{\omega_{\mathrm{p}}^{2}}{\omega(\omega+i \gamma)},
$$

where $\omega_{\mathrm{p}}$ is the bulk plasmon frequency, $\gamma$ is the relaxation constant of the bulk material, and $\epsilon_{\text {core }}(\omega)$ is the response of the bound electrons.

Under plane-wave illumination with a positive-frequency component of the form $\boldsymbol{E}_{\text {in }}^{+}=\tilde{\boldsymbol{E}}_{\text {in }}^{+} e^{-i \omega t}$, an oscillating dipole moment $\boldsymbol{d}_{\mathrm{n}}$ with a positive-frequency component of the form [3]

$$
\boldsymbol{d}_{\mathrm{n}}^{+}=\left(4 \pi \epsilon_{0} \epsilon_{\mathrm{b}}\right) r_{\mathrm{n}}^{3} \beta_{\mathrm{n}} \tilde{\boldsymbol{E}}_{\mathrm{in}}^{+} e^{-i \omega t}
$$

is induced in the nth MNP, where $\epsilon_{0}$ is the permittivity of free space and $\beta_{\mathrm{n}}$ is the Clausius Mossotti factor of the nth MNP in a bath of relative permittivity $\epsilon_{\mathrm{b}}$. The dipole moment $\boldsymbol{d}_{\mathrm{n}}$, the response field $\boldsymbol{E}_{\mathrm{n}}^{\text {res }}$ of which closely resembles that of a point dipole, can be retrieved as a combination of the positiveand negative-frequency components as $\boldsymbol{d}_{\mathrm{n}}=\boldsymbol{d}_{\mathrm{n}}^{+}+\boldsymbol{d}_{\mathrm{n}}^{-}$, where $\boldsymbol{d}_{\mathrm{n}}^{-}=\left(\boldsymbol{d}_{\mathrm{n}}^{+}\right)^{*}[3]$.

The dipole response field $\boldsymbol{E}_{\mathrm{n}}^{\mathrm{res}}$ emanated by the point dipole $\boldsymbol{d}_{\mathrm{n}}$ on the center of another MNP with radius $r_{\mathrm{j}}$, situated at a radial distance $R_{\mathrm{nj}}$, can be obtained as [49]

$$
\boldsymbol{E}_{\mathrm{n}}^{\mathrm{res}}=\frac{1}{\left(4 \pi \epsilon_{0} \epsilon_{\mathrm{b}}\right) R_{\mathrm{nj}}^{3}}\left[3\left(\boldsymbol{d}_{\mathrm{n}} \cdot \hat{\boldsymbol{s}}\right) \hat{\boldsymbol{s}}-\boldsymbol{d}_{\mathrm{n}}\right],
$$

where $\hat{\boldsymbol{s}}$ is the radial unit vector along the direction of the jth MNP from the center of the nth MNP. When $\boldsymbol{d}_{\mathrm{n}}$ is perpendicular (parallel) to the axis joining centers of the two MNPs, the above expression simplifies to

$$
\boldsymbol{E}_{\mathrm{n}}^{\mathrm{res}}=\frac{s_{\alpha} \boldsymbol{d}_{\mathrm{n}}}{\left(4 \pi \epsilon_{0} \epsilon_{\mathrm{b}}\right) R_{\mathrm{nj}}^{3}},
$$

where the orientation parameter $s_{\alpha}=-1$ (2) for perpendicular (parallel) polarization.

The external-field feedback dipole induced in the jth MNP due to the above dipole response field $\boldsymbol{E}_{\mathrm{n}}^{\text {res }}$ of the nth MNP can be obtained by using the positive-frequency component of (4) in (2) as

$$
\boldsymbol{d}_{\mathrm{j} \_\mathrm{n}}^{+}=\frac{\left(r_{\mathrm{j}}^{3} \beta_{\mathrm{j}} s_{\alpha}\right) \boldsymbol{d}_{\mathrm{n}}^{+}}{R_{\mathrm{nj}}^{3}} .
$$

In this study, we use the generalized nonlocal optical response (GNOR) theory [34,36] to model the Clausius Mossotti factor of an MNP with radius $r_{\mathrm{n}}$ as

$$
\beta_{\mathrm{n}}=\frac{\epsilon_{\mathrm{m}}(\omega)-\epsilon_{\mathrm{b}}\left(1+\delta_{\mathrm{NL}}\right)}{\epsilon_{\mathrm{m}}(\omega)+2 \epsilon_{\mathrm{b}}\left(1+\delta_{\mathrm{NL}}\right)},
$$

where the nonlocal correction $\delta_{\mathrm{NL}}$ is given by

$$
\delta_{\mathrm{NL}}=\frac{\epsilon_{\mathrm{m}}(\omega)-\epsilon_{\mathrm{core}}(\omega)}{\epsilon_{\text {core }}(\omega)} \frac{j_{1}\left(K_{\mathrm{L}} r_{\mathrm{n}}\right)}{K_{\mathrm{L}} r_{\mathrm{n}} j_{1}^{\prime}\left(K_{\mathrm{L}} r_{\mathrm{n}}\right)} .
$$

In the above equation, $j_{1}$ denotes the spherical Bessel function of the first kind of angular momentum order $1, j_{1}^{\prime}$ denotes its first-order differential with respect to the argument, and the longitudinal wave vector abides by the relationship $K_{\mathrm{L}}^{2}=$ $\epsilon_{\mathrm{m}}(\omega) / \xi^{2}(\omega)$. The bound electron response of the MNP is obtained as $\epsilon_{\text {core }}=\epsilon_{\text {expt }}(\omega)+\omega_{\mathrm{p}}^{2} /[\omega(\omega+i \gamma)]$ whereas the nonlocal parameter of the GNOR model is characterized by

$$
\xi^{2}(\omega)=\frac{\epsilon_{\text {core }}(\omega)\left[\kappa^{2}+\mathrm{D}(\gamma-i \omega)\right]}{\omega(\omega+i \gamma)},
$$

where $\mathrm{D}$ is the electron diffusion constant and $\kappa^{2}=$ $(3 / 5) v_{F}^{2}$ for $\omega \gg \gamma$ (in the high-frequency limit) where $v_{F}$ is the Fermi velocity of the MNP. It is evident that the Clausius Mossotti factor in the conventional local response approximation (LRA), given by the equation $\beta_{\mathrm{LRA}}=$ $\left[\epsilon_{\mathrm{m}}(\omega)-\epsilon_{\mathrm{b}}\right] /\left[\epsilon_{\mathrm{m}}(\omega)+2 \epsilon_{\mathrm{b}}\right][3]$, can be obtained by setting $\delta_{\mathrm{NL}} \rightarrow 0$ in $(6)$.

\section{B. External-field-induced plasmonic dipoles}

We now proceed to compute the effective external field experienced by the QE exciton in the presence of the MNP constellation. Under perpendicular illumination of the external field, all induced electric fields and dipole moment vectors lie parallel to the $z$ axis in the $x y$ plane. Thus, we will only be concerned with their values in the direction of $\hat{z}$.

Let us first consider the dipole moment component $d_{\mathrm{n}}$ directly induced in the nth MNP in the assembly, due to the perpendicularly incident external field. This can be obtained using (2) as

$$
d_{\mathrm{n}}=\left(4 \pi \epsilon_{0} \epsilon_{\mathrm{b}}\right) r_{\mathrm{n}}^{3} \beta_{\mathrm{n}} E_{0} e^{-i \omega t}+\text { c.c., for } \mathrm{n}=1,2, \ldots, N
$$

where c.c. denotes the complex conjugate of the preceding expression. As the total dipole moment induced in the nth MNP by the external field also comprises feedback via the surrounding MNPs, let us name (9) as its 0th level (or direct) external-field feedback dipole.

Using Eqs. (5) and (9) as outlined in Appendix A, we can find the positive-frequency component of the pth level external-field feedback dipole formed in the nth MNP due to the collective $(p-1)$ th level external-field feedback dipoles in the surrounding MNPs. It takes the following form:

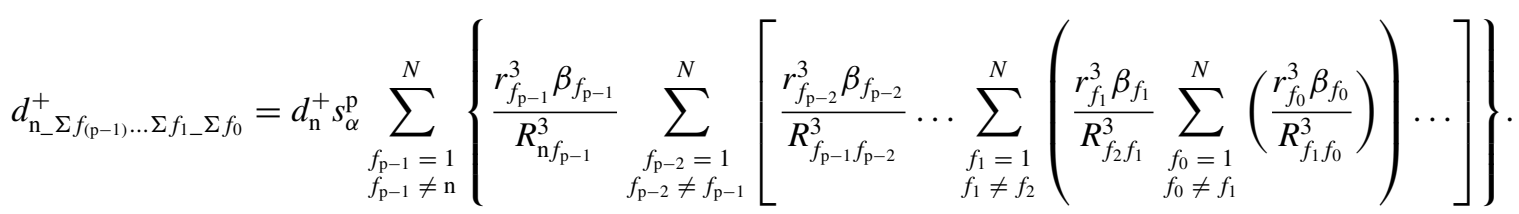


The nontruncated form of the total dipole moment induced in the nth MNP due to the external field and its feedback via surrounding MNPs, $d_{\mathrm{n} \_ \text {tot }}^{E}$, can be obtained as

$$
d_{\mathrm{n}_{-} \text {tot }}^{E}=d_{\mathrm{n}}^{+}\left(1+F_{\mathrm{n}_{-} E}\right)+\text { c.c., }
$$

where

$$
F_{\mathrm{n} \_E}=\frac{\sum_{\mathrm{p}=1}^{\infty} d_{\mathrm{n} \_\Sigma}^{+} f_{(\mathrm{p}-1) \ldots \Sigma} f_{1 \_} \Sigma f_{0}}{d_{\mathrm{n}}^{+}} .
$$

For the convergence of dipole feedback in this model, the pth level feedback dipole component arising due to the external field should possess a smaller absolute value than the respective $(\mathrm{p}-1)$ th level component. That is,

$$
\left|d_{\mathrm{n}_{-} \Sigma f_{(\mathrm{p}-1)} \ldots \Sigma f_{1 \_} \Sigma f_{0}}\right|<\left|d_{\mathrm{n} \_\Sigma f_{(\mathrm{p}-2)}^{+} \ldots \Sigma f_{1 \_} \Sigma f_{0}}\right|, \forall \mathrm{p} .
$$

\section{Quantum-emitter-induced plasmonic dipoles}

Due to exciton formation in the $\mathrm{QE}$, a transition dipole moment $d_{\mathrm{QE}}=\mu\left(\rho_{\mathrm{ge}}+\rho_{\mathrm{eg}}\right)$ is assumed to be induced at its center, where $\mu$ is the off-diagonal transition dipole matrix element (assumed real), $\rho_{\mathrm{ge}}=\tilde{\rho}_{\mathrm{ge}} e^{i \omega t}$ and $\rho_{\mathrm{eg}}=\tilde{\rho}_{\mathrm{eg}} e^{-i \omega t}$ denote the off-diagonal density matrix elements of the QE $[2,50]$. The positive-frequency component of the dipole moment directly induced in the nth MNP due to $d_{\mathrm{QE}}$ can be

$$
d_{\mathrm{n} \_\Sigma f_{(\mathrm{q}-1) \ldots \Sigma} \ldots f_{1} \Sigma \Sigma f_{0}}^{\mathrm{QE}+}=R_{\mathrm{n}}^{3} d_{\mathrm{n}}^{\mathrm{QEE}+} s_{\alpha}^{\mathrm{q}} \sum_{\substack{f_{\mathrm{q}-1}=1 \\ f_{\mathrm{q}-1} \neq \mathrm{n}}}^{N}\left\{\frac{r_{f_{\mathrm{q}-1}}^{3} \beta_{f_{\mathrm{q}-1}}}{R_{\mathrm{n} f_{\mathrm{q}-1}}^{3}} \sum_{\substack{f_{\mathrm{q}-2}=1 \\ f_{\mathrm{q}-2} \neq f_{\mathrm{q}-1}}}^{N}\right.
$$$$
\left.\left[\frac{r_{f_{\mathrm{q}-2}}^{3} \beta_{f_{\mathrm{q}-2}}}{R_{f_{\mathrm{q}-1} f_{\mathrm{q}-2}}^{3}} \ldots \sum_{\substack{f_{1}=1 \\ f_{1} \neq f_{2}}}^{N}\left(\frac{r_{f_{1}}^{3} \beta_{f_{1}}}{R_{f_{2} f_{1}}^{3}} \sum_{\substack{f_{0}=1 \\ f_{0} \neq f_{1}}}^{N}\left(\frac{r_{f_{0}}^{3} \beta_{f_{0}}}{R_{f_{1} f_{0}}^{3} R_{f_{0}}^{3}}\right)\right) \ldots\right]\right\}
$$

obtained with the aid of (5) as

$$
d_{\mathrm{n}}^{\mathrm{QE}+}=\frac{\left(s_{\alpha} r_{\mathrm{n}}^{3} \beta_{\mathrm{n}}\right) \mu \tilde{\rho}_{\mathrm{eg}} e^{-i \omega t}}{\epsilon_{\mathrm{effS}} R_{\mathrm{n}}^{3}},
$$

where $\epsilon_{\mathrm{effS}}=\left(2 \epsilon_{\mathrm{b}}+\epsilon_{\mathrm{s}}\right) /\left(3 \epsilon_{\mathrm{b}}\right)$ accounts for the screening of the emanating field due to the QE dielectric [1,2,32]. Equation (14) denotes the 0th level feedback dipole formed in the nth MNP due to the QE.

The positive-frequency component of the first-level QE feedback dipole formed in the nth MNP due to the collective Oth level QE feedback dipoles in the surrounding MNPs (indexed using $f_{0}$ where $f_{0} \neq \mathrm{n}$ ) can be obtained using (14) and (5) as

$$
d_{\mathrm{n} \_\Sigma f_{0}}^{\mathrm{QE}+}=\frac{\left(s_{\alpha}^{2} r_{\mathrm{n}}^{3} \beta_{\mathrm{n}}\right) \mu \tilde{\rho}_{\mathrm{eg}} e^{-i \omega t}}{\epsilon_{\mathrm{effS}}} \sum_{\substack{f_{0}=1 \\ f_{0} \neq \mathrm{n}}}^{N}\left(\frac{r_{f_{0}}^{3} \beta_{f_{0}}}{R_{\mathrm{n} f_{0}}^{3} R_{f_{0}}^{3}}\right) .
$$

By repeating this procedure as outlined in the earlier section, we can obtain the qth level QE feedback dipole formed in the nth MNP due to the collective $(q-1)$ th level feedback dipoles in the surrounding MNPs (indexed using the subscript $f_{q-1}$ where $f_{q-1} \neq \mathrm{n}$ ) as
Similar to the external-field-induced case, the nontruncated form of the total dipole moment induced in the nth MNP due to the $\mathrm{QE}$ and its feedback via the surrounding MNPs $\left(d_{\mathrm{n} \_t \text { tot }}^{\mathrm{QE}}\right)$ can be obtained as

$$
d_{\mathrm{n}_{-} \text {tot }}^{\mathrm{QE}}=d_{\mathrm{n}}^{\mathrm{QE}+}\left(1+F_{\mathrm{n} \_\mathrm{QE}}\right)+\text { c.c. },
$$

where

$$
F_{\mathrm{n} \_\mathrm{QE}}=\frac{\sum_{\mathrm{q}=1}^{\infty} d_{\mathrm{n} \_\Sigma f_{(\mathrm{q}-1) \ldots \Sigma}}^{\mathrm{QE}+}}{d_{\mathrm{n}}^{\mathrm{QE}+}},
$$

and the QE feedback convergence requires

$$
\left|d_{\mathrm{n}_{-} \Sigma f_{(\mathrm{q}-1)} \ldots \Sigma f_{1 \_} \Sigma f_{0}}^{\mathrm{QE}+}\right|<\left|d_{\mathrm{n} \_\Sigma f_{(\mathrm{q}-2)} \ldots \Sigma f_{1 \_} \Sigma f_{0}}^{\mathrm{QE}+}\right|, \forall \mathrm{q} .
$$

\section{Effective field experienced by the QE exciton}

The total dipole moment $d_{\text {n_tot }}$ induced in the nth MNP in our constellation can be found, while accounting for the infinite series of feedback via the other MNPs, as

$$
d_{\mathrm{n} \_ \text {tot }}=d_{\mathrm{n}_{-} \text {tot }}^{E}+d_{\mathrm{n} \_ \text {tot }}^{\mathrm{QE}} \text {. }
$$

We can then calculate the total electric field incident on the QE exciton, accounting for both the external field $E$ and the collective MNP dipole response fields using (20) and (3) as

$$
E_{\mathrm{QE}} \approx \frac{1}{\epsilon_{\mathrm{effS}}}\left(E+\sum_{\mathrm{n}=1}^{N} \frac{s_{\alpha} d_{\mathrm{n} \_ \text {tot }}}{\left(4 \pi \epsilon_{0} \epsilon_{\mathrm{b}}\right) R_{\mathrm{n}}^{3}}\right) .
$$

The resultant Rabi frequency $\left(\Omega^{r}\right)$ experienced by the QE exciton is obtainable using the above equation, where $E_{\mathrm{QE}}=$ $\tilde{E}_{\mathrm{QE}}^{+} e^{-i \omega t}+$ c.c. as

$$
\Omega^{r}=\mu \tilde{E}_{\mathrm{QE}}^{+} / \hbar=\Omega+\eta \tilde{\rho}_{\mathrm{eg}} .
$$

We can obtain the Rabi frequency in the absence of quantum coherences $(\Omega)$ and the QE self-interaction coefficient $(\eta)$ using (20), (21), and (22) as

$$
\begin{gathered}
\Omega=\Omega^{0}\left\{1+\sum_{\mathrm{n}=1}^{N}\left[\frac{s_{\alpha} r_{\mathrm{n}}^{3} \beta_{\mathrm{n}}\left(1+F_{\mathrm{n} \_E}\right)}{R_{\mathrm{n}}^{3}}\right]\right\}, \\
\eta=\frac{s_{\alpha}^{2} \mu^{2}}{\left(4 \pi \epsilon_{0} \epsilon_{\mathrm{b}}\right) \hbar \epsilon_{\mathrm{effS}}^{2}} \sum_{\mathrm{n}=1}^{N}\left[\frac{r_{\mathrm{n}}^{3} \beta_{\mathrm{n}}\left(1+F_{\mathrm{n} \_\mathrm{QE}}\right)}{R_{\mathrm{n}}^{6}}\right],
\end{gathered}
$$

where the Rabi frequency experienced by the isolated exciton in the external field $E$ (when $N=0$ or $R \rightarrow \infty$ ) is denoted by $\Omega^{0}=\mu E_{0} /\left(\hbar \epsilon_{\text {effS }}\right)$ [32]. Notice that the normalized Rabi frequency for the case of single MNP-QE nanohybrid [2,32] can be retrieved from the above equations by setting $N=1$, where $F_{\mathrm{n}_{-} E}=F_{\mathrm{n} \_\mathrm{QE}}=0$.

\section{E. Symmetric constellation}

In the earlier section, we derived the resultant Rabi frequency experienced by a quantum emitter surrounded by a generic planar MNP constellation which was not necessarily 
symmetric. Let us now reduce the derived equations for a symmetric setup where the QE lies at the center of a circular constellation of $N$ identical, equidistant MNPs.

Consider a symmetric version of the setup presented in Fig. 1 where $\forall \mathrm{n} \in\{1,2, \ldots, N\}$, radius $r_{\mathrm{n}}=r$, polarizability $\beta_{\mathrm{n}}=\beta$, MNP-QE center separation $R_{\mathrm{n}}=R$, and the summation of the cube of distances (center separations) from the $n$th MNP to all other MNPs (indexed in the summation as $\mathrm{j}$ where $\mathrm{j} \neq \mathrm{n}$ ) is commonly given by

$$
\zeta=\sum_{\substack{\mathrm{j}=1 \\ \mathrm{j} \neq \mathrm{n}}}^{N}\left(\frac{1}{R_{\mathrm{nj}}^{3}}\right),
$$

which will be constant for a given setup due to the circular symmetry. For $N=1, \zeta=0$.

Notice that for such symmetric setups, the positivefrequency components of the pth level feedback dipole formed in the nth MNP due to the external field, given by Eq. (10), and the qth level feedback dipole formed due to the QE, given by Eq. (16), simplify to their symmetric versions

$$
\begin{aligned}
& {\left[d_{\mathrm{n} \_\Sigma f_{(\mathrm{p}-1)} \ldots \Sigma f_{1-} \Sigma f_{0}}^{+}\right]_{\mathrm{sym}}=d_{\mathrm{n}}^{+}\left(s_{\alpha} r^{3} \beta \zeta\right)^{\mathrm{p}},} \\
& {\left[d_{\mathrm{n} \_\Sigma f_{(\mathrm{q}-1)} \ldots \Sigma f_{1 \_} \Sigma f_{0}}^{\mathrm{QE}+}\right]_{\mathrm{sym}}=d_{\mathrm{n}}^{\mathrm{QE}+}\left(s_{\alpha} r^{3} \beta \zeta\right)^{\mathrm{q}} .}
\end{aligned}
$$

Thus, $F_{\mathrm{n}_{-} E}$ and $F_{\mathrm{n} \_ \text {QE }}$ reduce to their symmetric versions

$$
\begin{gathered}
{\left[F_{\mathrm{n}_{-} E}\right]_{\mathrm{sym}}=\sum_{\mathrm{p}=1}^{\infty}\left(s_{\alpha} r^{3} \beta \zeta\right)^{\mathrm{p}},} \\
{\left[F_{\mathrm{n}_{-} \mathrm{QE}}\right]_{\text {sym }}=\sum_{\mathrm{q}=1}^{\infty}\left(s_{\alpha} r^{3} \beta \zeta\right)^{\mathrm{q}} .}
\end{gathered}
$$

As they both represent infinite complex geometric series, the summations can be simplified to

$$
\left[F_{\mathrm{n} \_E}\right]_{\mathrm{sym}}=\left[F_{\mathrm{n} \_\mathrm{QE}}\right]_{\mathrm{sym}}=\frac{s_{\alpha} r^{3} \beta \zeta}{1-s_{\alpha} r^{3} \beta \zeta},
$$

with the convergence condition

$$
\left|s_{\alpha} r^{3} \beta \zeta\right|<1
$$

which determines the usability of this model.

Using (20) and (28), the positive-frequency component of the total dipole moment experienced by the nth MNP in a symmetric setup can be obtained as

$$
\left[d_{\mathrm{n} \_ \text {tot }}^{+}\right]_{\mathrm{sym}}=\frac{d_{\mathrm{n}}^{+}+d_{\mathrm{n}}^{\mathrm{QE}+}}{1-s_{\alpha} r^{3} \beta \zeta} .
$$

By substituting $\left[d_{\mathrm{n}_{-} \text {tot }}^{+}\right]_{\mathrm{sym}}$ in place of $d_{\mathrm{n}_{-} \text {tot }}^{+}$in Eq. (21), and by setting $R_{\mathrm{n}}=R$, we can extract the slowly varying amplitude of the total electric field experienced by a QE exciton situated at the center of our symmetric MNP ring as

$$
\left[\tilde{E}_{\mathrm{QE}}^{+}\right]_{\mathrm{sym}}=\frac{\hbar}{\mu}\left[\Omega^{r}\right]_{\mathrm{sym}}=\frac{\hbar}{\mu}\left([\Omega]_{\mathrm{sym}}+[\eta]_{\mathrm{sym}} \tilde{\rho}_{\mathrm{eg}}\right)
$$

where

$$
\begin{aligned}
& {[\Omega]_{\mathrm{sym}}=\Omega^{0}\left\{1+\frac{N s_{\alpha} r^{3} \beta}{R^{3}}\left(\frac{1}{1-s_{\alpha} r^{3} \beta \zeta}\right)\right\},} \\
& {[\eta]_{\mathrm{sym}}=\frac{N s_{\alpha}^{2} r^{3} \mu^{2} \beta}{\left(4 \pi \epsilon_{0} \epsilon_{\mathrm{b}}\right) R^{6} \hbar \epsilon_{\mathrm{effS}}^{2}}\left(\frac{1}{1-s_{\alpha} r^{3} \beta \zeta}\right) .}
\end{aligned}
$$

\section{F. Open quantum system}

We treat the excitonic system at the center of the QE quantum mechanically as a two-level atom, using the density matrix formalism [51]. The Hamiltonian of the two-level atomic system under the influence of the MNP assembly and the externally applied electric field can be obtained as [32,35,52]

$$
\hat{\mathcal{H}}=\hbar \omega_{0} \hat{\sigma}^{+} \hat{\sigma}^{-}-\mu E_{\mathrm{QE}}\left(\hat{\sigma}^{+}+\hat{\sigma}^{-}\right),
$$

where $\hat{\sigma}^{-}=|g\rangle\left\langle e\left|, \hat{\sigma}^{+}=\right| e\right\rangle\langle g|| g\rangle=,(1,0)^{\mathrm{T}}$, and $|e\rangle=$ $(0,1)^{\mathrm{T}}$ denote the exciton annihilation and creation operators, and the atomic ground and excited states, respectively. The above Hamiltonian, when taken in isolation describes a closed quantum system where the impact of the environment is not yet taken into consideration. It couples with the environment to form an open quantum system exhibiting irreversible dynamics that can be accounted for using Lindblad terms in the master equation of the QE density matrix $\hat{\rho}$ as follows $[2,30,32]$ :

$$
\dot{\hat{\rho}}=\frac{i}{\hbar}[\hat{\rho}, \hat{\mathcal{H}}]+\lambda_{1} \mathcal{L}_{\hat{\sigma}^{-}}+\lambda_{2} \mathcal{L}_{\hat{\sigma}^{+}}+\lambda_{3} \mathcal{L}_{\hat{\sigma}^{+} \hat{\sigma}^{-}} .
$$

The three Lindblad terms $\lambda_{1} \mathcal{L}_{\hat{\sigma}^{-}}, \lambda_{2} \mathcal{L}_{\hat{\sigma}^{+}}$, and $\lambda_{3} \mathcal{L}_{\hat{\sigma}^{+} \hat{\sigma}^{-}}$represent bath-induced decay of the two-level atomic system from the excited to ground state, bath-induced excitation vice versa, and elastic scattering processes between the bath and the quantum system, respectively. Their expansion takes the form

$$
\mathcal{L}_{\hat{A}}=2 \hat{A} \hat{\rho} \hat{A}^{\dagger}-\hat{A}^{\dagger} \hat{A} \hat{\rho}-\hat{\rho} \hat{A}^{\dagger} \hat{A} .
$$

For optical frequencies, even near room temperature, $\lambda_{2} \approx$ 0 [2]. Let,

$$
\begin{aligned}
& T_{1}=1 /\left(2 \lambda_{1}\right), \\
& T_{2}=1 /\left(\lambda_{1}+\lambda_{3}\right),
\end{aligned}
$$

where $T_{1}$ is the energy or population relaxation time of the $\mathrm{QE}$ which leads to a mixing between (populations or the diagonal density matrix elements) $\rho_{\mathrm{gg}}$ and $\rho_{\mathrm{ee}}[2] . T_{2}$ is the polarization relaxation or dephasing time which causes losses in the off-diagonal density matrix elements $[21,53]$. With these definitions, the matrix form of the master equation (34) in the basis space $\{|g\rangle,|e\rangle\}$ reads as [2,32]

$$
\dot{\hat{\rho}}=\frac{i}{\hbar}\left[\begin{array}{cc}
-\mu E_{\mathrm{QE}}\left(\rho_{\mathrm{ge}}-\rho_{\mathrm{eg}}\right) & -\mu E_{\mathrm{QE}}\left(\rho_{\mathrm{gg}}-\rho_{\mathrm{ee}}\right)+\hbar \omega_{0} \rho_{\mathrm{ge}} \\
-\mu E_{\mathrm{QE}}\left(\rho_{\mathrm{ee}}-\rho_{\mathrm{gg}}\right)-\hbar \omega_{0} \rho_{\mathrm{eg}} & -\mu E_{\mathrm{QE}}\left(\rho_{\mathrm{eg}}-\rho_{\mathrm{ge}}\right)
\end{array}\right]-\left[\begin{array}{cc}
\left(\rho_{\mathrm{gg}}-1\right) / T_{1} & \rho_{\mathrm{ge}} / T_{2} \\
\rho_{\mathrm{eg}} / T_{2} & \rho_{\mathrm{ee}} / T_{1}
\end{array}\right] .
$$


By solving this, we can analyze the behavior of the two-level excitonic system under the influence of the MNP assembly and the external illumination.

\section{G. Steady-state analysis}

In this section, we summarize the approach outlined in [32] to obtain the steady-state solution of the QE master equation for completeness. Using elementwise comparison of the leftand right-hand sides of (37) and the definition of effective Rabi frequency $\Omega^{r}=\mu \tilde{E}_{\mathrm{QE}}^{+} / \hbar$, we can arrive at the following Bloch equations for the two-level excitonic system:

$$
\begin{aligned}
& \dot{\rho}_{\mathrm{ee}}=-\frac{\rho_{\mathrm{ee}}}{T_{1}}+i \Omega^{r} \tilde{\rho}_{\mathrm{ge}}-i \Omega^{r *} \tilde{\rho}_{\mathrm{eg}}, \\
& \dot{\rho}_{\mathrm{gg}}=\frac{\rho_{\mathrm{ee}}}{T_{1}}-i \Omega^{r} \tilde{\rho}_{\mathrm{ge}}+i \Omega^{r *} \tilde{\rho}_{\mathrm{eg}}, \\
& \dot{\tilde{\rho}}_{\mathrm{eg}}=-\left[i\left(\omega_{0}-\omega\right)+1 / T_{2}\right] \tilde{\rho}_{\mathrm{eg}}+i \Omega^{r} \Delta,
\end{aligned}
$$

where $\Delta=\rho_{\mathrm{gg}}-\rho_{\mathrm{ee}}$ denotes the population difference. Defining the real-imaginary separations $\tilde{\rho}_{\mathrm{ge}}=\mathcal{A}+i \mathcal{B}, \tilde{\rho}_{\mathrm{eg}}=$ $\mathcal{A}-i \mathcal{B}, \Omega=\Omega_{\mathrm{re}}+i \Omega_{\mathrm{im}}$, and $\eta=\eta_{\mathrm{re}}+i \eta_{\text {im }}$, we can recast (38) to the following form $[2,32]$ :

$$
\begin{aligned}
& \dot{\mathcal{A}}=-\frac{\mathcal{A}}{T_{2}}+\delta \mathcal{B}-\left(\Omega_{\mathrm{im}}+\eta_{\mathrm{im}} \mathcal{A}-\eta_{\mathrm{re}} \mathcal{B}\right) \Delta, \\
& \dot{\mathcal{B}}=-\frac{\mathcal{B}}{T_{2}}-\delta \mathcal{A}-\left(\Omega_{\mathrm{re}}+\eta_{\mathrm{re}} \mathcal{A}+\eta_{\mathrm{im}} \mathcal{B}\right) \Delta, \\
& \dot{\Delta}=\frac{1-\Delta}{T_{1}}+4\left[\Omega_{\mathrm{im}} \mathcal{A}+\Omega_{\mathrm{re}} \mathcal{B}+\eta_{\mathrm{im}}\left(\mathcal{A}^{2}+\mathcal{B}^{2}\right)\right],
\end{aligned}
$$

where the detuning is denoted by $\delta=\omega-\omega_{0}$. In the steady state where $\dot{\mathcal{A}}=\dot{\mathcal{B}}=0$, we can manipulate (39a) and (39b) to obtain [32]

$$
\begin{aligned}
& \mathcal{A}=-\operatorname{Re}\left(\frac{\Omega \Delta}{\delta+\eta \Delta+i / T_{2}}\right), \\
& \mathcal{B}=\operatorname{Im}\left(\frac{\Omega \Delta}{\delta+\eta \Delta+i / T_{2}}\right) .
\end{aligned}
$$

By substituting (40) in (39c) and setting $\dot{\Delta}=0$, we can obtain the following steady-state equation for the QE population difference $\Delta$ :

$$
\Delta^{3}+\bar{w}_{2} \Delta^{2}+\bar{w}_{1} \Delta+\bar{w}_{0}=0
$$

where

$$
\begin{aligned}
& \bar{w}_{2}=\frac{2 T_{2}^{2} \delta \eta_{\mathrm{re}}+2 T_{2} \eta_{\mathrm{im}}-T_{2}^{2}\left(\eta_{\mathrm{re}}^{2}+\eta_{\mathrm{im}}^{2}\right)}{T_{2}^{2}\left(\eta_{\mathrm{re}}^{2}+\eta_{\mathrm{im}}^{2}\right)}, \\
& \bar{w}_{1}=\frac{T_{2}\left(4 T_{1}|\Omega|^{2}-2 \eta_{\mathrm{im}}\right)+T_{2}^{2}\left(\delta^{2}-2 \delta \eta_{\mathrm{re}}\right)+1}{T_{2}^{2}\left(\eta_{\mathrm{re}}^{2}+\eta_{\mathrm{im}}^{2}\right)}, \\
& \bar{w}_{0}=\frac{-T_{2}^{2} \delta^{2}-1}{T_{2}^{2}\left(\eta_{\mathrm{re}}^{2}+\eta_{\mathrm{im}}^{2}\right)} .
\end{aligned}
$$

By solving (41) for $-1 \leqslant \Delta \leqslant 1$ we can obtain the $\mathrm{QE}$ population difference, substitution of which in (40) yields $\mathcal{A}$ and $\mathcal{B}$, and hence the off-diagonal density matrix elements $\rho_{\mathrm{eg}}$ and $\rho_{\mathrm{ge}}$.

Let us now look at how the obtained solutions for the density matrix elements can be used to analyze the steady-state behavior of the excitonic system. First, the energy absorption rate of the $\mathrm{QE}$ can be computed as [2]

$$
Q_{\mathrm{QE}}=\hbar \omega_{0} \rho_{e e} / T_{1}=\hbar \omega_{0}(1-\Delta) /\left(2 T_{1}\right) .
$$

Second, we recast the $\mathrm{QE}$ Bloch equation (38c) in the following form:

$$
\dot{\tilde{\rho}}_{\mathrm{eg}}=-[i(\Pi-\omega)+\Lambda] \tilde{\rho}_{\mathrm{eg}}+i \Omega \Delta,
$$

where

$$
\begin{aligned}
& \Pi=\omega_{0}-\eta_{\mathrm{re}} \Delta, \\
& \Lambda=1 / T_{2}+\eta_{\mathrm{im}} \Delta
\end{aligned}
$$

denote the effective energy and dephasing rate of the $\mathrm{QE}$ excitonic transition under the influence of the neighboring MNP assembly [33]. Let us call the two factors $\Pi_{f}=\eta_{\mathrm{re}} \Delta$ and $\Lambda_{f}=\eta_{\mathrm{im}} \Delta$ as the exciton transition energy (red)shift and the dephasing rate (blue)shift, respectively [32].

We conclude the formalism section recalling that all the above equations can be converted to their LRA based forms by setting $\delta_{\mathrm{NL}} \rightarrow 0$ as mentioned in Sec. II A. Moreover, the time-averaged energy absorption rate of the nth MNP in the symmetric setup can be estimated as (see Appendix B for a detailed derivation)

$$
\begin{aligned}
Q_{\mathrm{n}}= & \left\{\frac{\left(4 \pi \epsilon_{0} \epsilon_{\mathrm{b}}\right) r^{3}(i \omega) \beta_{\mathrm{LRA}}^{*}}{\epsilon_{\mathrm{effM}}} \mid\left(E_{0}+\frac{s_{\alpha} \mu \tilde{\rho}_{\mathrm{ge}}}{\left(4 \pi \epsilon_{0} \epsilon_{\mathrm{b}}\right) \epsilon_{\mathrm{effS}} R^{3}}\right)\right. \\
& \left.\times\left.\frac{1}{\left(1-s_{\alpha} r^{3} \beta_{\mathrm{LRA}}^{*} \zeta\right)}\right|^{2}\right\}+ \text { c.c., }
\end{aligned}
$$

where $\epsilon_{\mathrm{effM}}=\left(2 \epsilon_{\mathrm{b}}+\epsilon_{m}\right) /\left(3 \epsilon_{\mathrm{b}}\right)$ and the total-energy absorption rate of the entire symmetric MNP-QE constellation can thus be obtained as

$$
Q_{\mathrm{tot}} \approx N Q_{\mathrm{n}}+Q_{\mathrm{QE}}
$$

\section{RESULTS AND DISCUSSION}

In this section, we study the behavior of coherently illuminated QEs under the influence of planar, symmetric MNP assemblies using the presented analytical equations. We focus our attention on small MNPs where nonlocal effects are prominent and comparatively large inter-MNP distances within the quasistatic limit where hybridization of the dipole mode of one MNP with higher-order multipoles of a neighboring pair (resulting in additional higher-order terms to the plasmon coupling [54]) can be safely neglected.

The common parameters used for the presented analysis are as follows: electric field strength of the external illumination $E_{0}=1 \times 10^{5} \mathrm{~V} \mathrm{~m}^{-1}$, orientation parameter $s_{\alpha}=-1$ (perpendicular illumination), polarization relaxation (dephasing) time of the isolated QE $T_{2}=0.3 \mathrm{~ns}$, energy or population relaxation time of the isolated $\mathrm{QE} T_{1}=0.8 \mathrm{~ns}$ [2], dielectric constant of the QD material $\epsilon_{\mathrm{s}}=6$ and QE dipole moment $\mu=2$ e nm [2]. Our analysis uses an assembly of gold MNPs, each with radius $r=3 \mathrm{~nm}$, bulk plasma frequency $\hbar \omega_{\mathrm{p}}=9.02 \mathrm{eV}$, bulk damping rate $\hbar \gamma=0.071 \mathrm{eV}$, Fermi velocity $v_{f}=1.39 \times 10^{6} \mathrm{~m} \mathrm{~s}^{-1}$, and diffusion constant $\mathrm{D} \approx$ 
$8.62 \times 10^{-4} \mathrm{~m}^{2} \mathrm{~s}^{-1}$ [34]. The experimentally measured bulk dielectric data $\epsilon_{\text {expt }}$ for gold are obtained using the tabulations by Johnson and Christy [55].

In our analysis, we vary the number of MNPs $N$ in our planar-symmetric constellation from 1 to 6 to obtain the presented graphical results, while utilizing the symmetry of the hybrid nanosystem to calculate the inter-MNP distances $\left(R_{\mathrm{nk}}\right)$, in terms of the center separation between each MNP and QE $(R)$. We list these distances below, in the notations used in our analytical equations, for the convenience of the readers:

$$
\begin{aligned}
& \text { For all } \mathrm{n}, \mathrm{k}, R_{\mathrm{nn}}=0 \text { and } R_{\mathrm{nk}}=R_{\mathrm{kn}} \text {, } \\
& N=2 \Rightarrow R_{12}=2 R \text {, } \\
& N=3 \Rightarrow R_{12}=R_{13}=R_{23}=\sqrt{3} R, \\
& N=4 \Rightarrow R_{12}=R_{14}=R_{23}=R_{34}=\sqrt{2} R \text {, } \\
& R_{13}=R_{24}=2 R \text {, } \\
& N=5 \Rightarrow R_{12}=R_{23}=R_{34}=R_{45}=R_{15}=2 R \sin \frac{\pi}{5} \text {, } \\
& R_{13}=R_{14}=R_{24}=R_{25}=R_{35}=2 R_{12} \sin \frac{3 \pi}{10}, \\
& N=6 \Rightarrow R_{12}=R_{23}=R_{34}=R_{45}=R_{56}=R_{16}=R \text {, } \\
& R_{13}=R_{24}=R_{35}=R_{46}=R_{15}=R_{26}=\sqrt{3} R, \\
& R_{14}=R_{25}=R_{36}=2 R \text {. }
\end{aligned}
$$

Thus, it is evident that we can obtain the inter-MNP distances for one side of a symmetric setup as $2 R \sin (k \pi / N)$ where $k=1,2, \ldots,\lfloor N / 2\rfloor$ up to and beyond $N=6$. If one intends to use $N>6$, it is worth noticing that the center separation between adjacent MNPs will be less than $R$ for a symmetric setup. The distances for such assemblies should be cautiously chosen when using this model to stay within the quasistatic limit while maintaining weak inter-MNP coupling. A surface-separation/diameter ratio at least $\geqslant 1$ between adjacent gold MNPs is consistent with weak near-field plasmon coupling [48] where our model is applicable.

Throughout this section, we use the GNOR model to characterize the optical polarizability $\beta$ of the gold nanoparticles, except where we explicitly mention the use of the LRA model for comparison purposes. In Fig. 2, we have illustrated the variation of GNOR based $|\beta|$ for three different (relative) permittivity values of submerging media, $\epsilon_{\mathrm{b}}=1.78,3.3$, and 5.45. It is useful to notice that the plasmonic peak amplifies and redshifts with increasing submerging medium permittivity. We have also depicted the variation of $|\beta|$ as predicted by the conventional LRA model for $\epsilon_{\mathrm{b}}=3.3$, which is seen to possess a larger, redshifted peak compared to its nonlocal counterpart, as is also suggested by literature [34].

\section{A. Narrow-band analysis}

Let us first analyze the optical response of a QE placed at the center of an equispaced, planar MNP ring, when the external coherent illumination sweeps a narrow frequency band of $5 \mathrm{meV}$ around the QE resonance. Figure 3 depicts

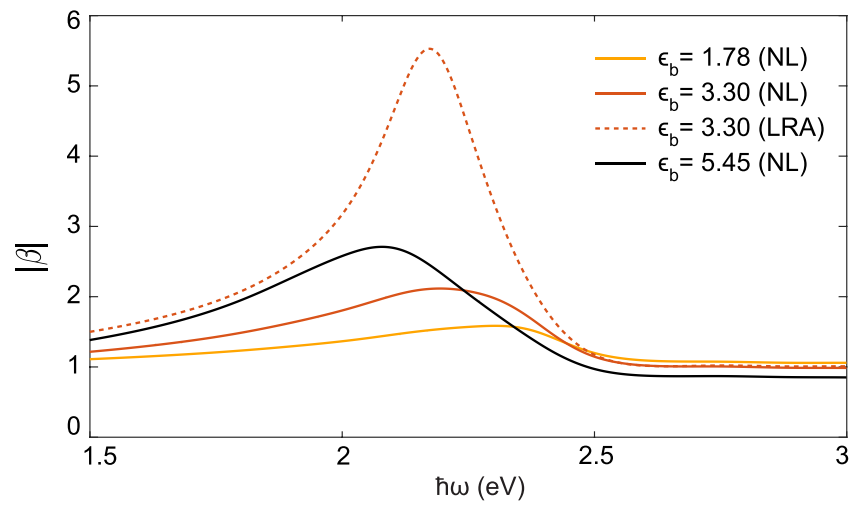

FIG. 2. Variation of the absolute polarizability $(|\beta|)$ for a gold nanoparticle of radius $3 \mathrm{~nm}$ as predicted by the nonlocal GNOR (NL) model at submerging medium (relative) permittivities $\epsilon_{\mathrm{b}}=1.78,3.3$, 5.45 , and by the local response approximation (LRA) model at $\epsilon_{\mathrm{b}}=$ 3.3 .

such sample plots we have obtained for $\mathrm{QE}$ absorption rate $Q_{\mathrm{QE}}$, population difference $\Delta$, and the real $(\mathcal{A})$ and imaginary $(\mathcal{B})$ parts of the slowly varying off-diagonal density matrix element component $\left(\tilde{\rho}_{\mathrm{ge}}=\mathcal{A}+i \mathcal{B}\right)$, for a system with bare excitonic resonance energy $\hbar \omega_{0}=2 \mathrm{eV}$, submerging medium permittivity $\epsilon_{\mathrm{b}}=5.45$ and MNP-QE center separation $R=$ $15 \mathrm{~nm}$. Notice (using Fig. 2) that for these sample plots, the bare excitonic resonance energy lies close to the plasmonic resonance energy of a 3 -nm radius MNP at $\epsilon_{\mathrm{b}}=5.45\left(\hbar \omega_{\mathrm{sp}} \approx\right.$ $2.09 \mathrm{eV})$.

Figure 3(b), which depicts the variation of the QE population difference $\Delta=\rho_{\mathrm{gg}}-\rho_{\mathrm{ee}}$ shows that $\Delta \rightarrow 1$ as the absolute detuning of the incident field frequency from the $\mathrm{QE}$ resonance gets larger, for all values of $\mathrm{N}$. Moreover, $\Delta \rightarrow 0$ when the detuning $\rightarrow 0$ (that is, when $\omega \rightarrow \omega_{0}$ ), tracing a singly dipped spectral shape. As is also suggested by (43), QE absorption rate depicted in Fig. 3(a) follows a singly peaked spectral shape with its peak aligning with the dip of $\Delta$ at resonance, and tending to zero as the detuning increases. Also note from Figs. 3(c) and 3(d) that $\mathcal{A}$ and $\mathcal{B}$ possess Fano-type line shapes around QE resonance which tend to zero with increasing detuning, for all values of $N$ considered.

We examined a range of $Q_{\mathrm{QE}}, \Delta, \mathcal{A}$, and $\mathcal{B}$ plots in a wide parameter region where $\epsilon_{\mathrm{b}}$ was varied from $1.78-5.45$ and $\hbar \omega_{0}$ was varied from $1.5-3 \mathrm{eV}$. In regions of reduced plasmonic impact on the QE, resulting from parameters/parameter combinations such as high detunings of $\omega_{0}$ from the plasmonic resonance $\left(\omega_{\mathrm{sp}}\right)$, low $\epsilon_{\mathrm{b}}$ (resulting in diminishing plasmonic peaks as depicted in Fig. 2), or large values of $R$, the amplitudes of both the Fano peak and trough of $\mathcal{B}$ was seen to reduce, accompanied by distortions to the Fano shape with higher trough amplitudes in comparison to the respective peaks. In the entire parameter space examined, the singly peaked, singly dipped, and Fano-type line shapes around QE resonance observed for $Q_{\mathrm{QE}}, \Delta$, and $\mathcal{A}$, respectively, were preserved (similar to the sample plots in Fig. 3). However, meV scale linewidth variations were observable for $Q_{\mathrm{QE}}$ and $\Delta$, as shown in the inset in Fig. 3(a). We analyze these linewidth variations in the next section. 

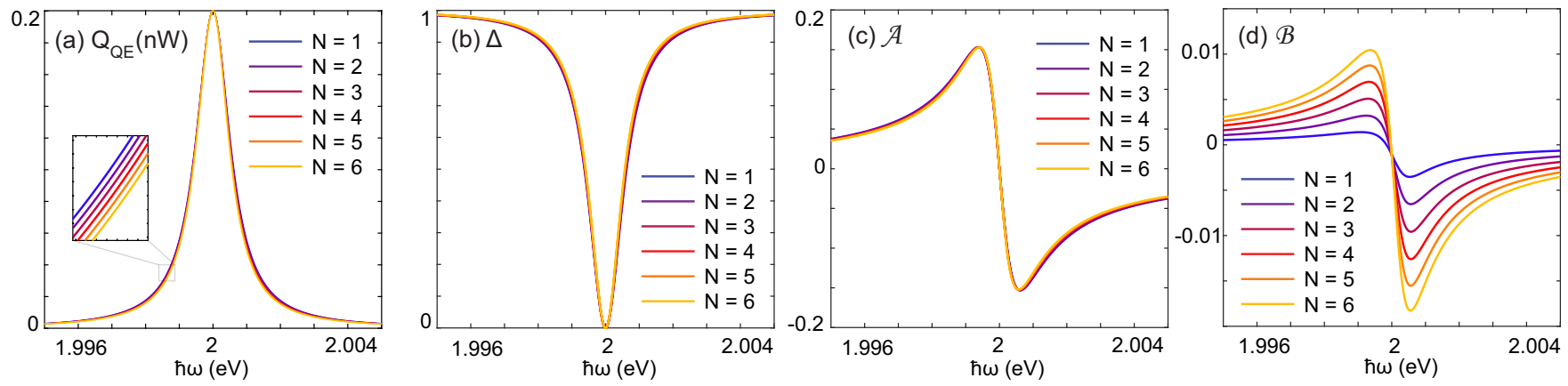

FIG. 3. Sample plots depicting the variation of (a) QE absorption rate $Q_{\mathrm{QE}}$, (b) QE population difference $\Delta$, (c) real and (d) imaginary parts of the slowly time-varying off-diagonal density matrix element component $\tilde{\rho}_{\mathrm{ge}}=\mathcal{A}+i \mathcal{B}$ (where $\rho_{\mathrm{ge}}=\tilde{\rho}_{\mathrm{ge}} e^{i \omega t}$ ) under the influence of $N=1, \ldots, 6 \mathrm{MNPs}$, plotted against the energy $\hbar \omega$ of the coherent external illumination in a narrow band of 5 meV around the QE resonance $\hbar \omega_{0}$. For these sample plots, the $\mathrm{QE}$ resonance $\hbar \omega_{0}=2 \mathrm{eV}$, submerging medium permittivity $\epsilon_{\mathrm{b}}=5.45$ and the distance from QE to all MNPs, $R=15 \mathrm{~nm}$.

\section{B. Analysis of linewidth variations}

We analyzed, using both nonlocal GNOR (NL) and local response approximation (LRA) based models, the variation of the full width at half-maximum (FWHM) of $Q_{\mathrm{QE}}$ against the bare excitonic resonance energy $\left(\hbar \omega_{0}\right)$ and MNP-QE center separation $(R)$ for a number of cases, where the submerging medium permittivity $\epsilon_{\mathrm{b}}$ was varied from $1.78-5.45$. For all cases within the aforementioned parameter range, the convergence factor lay below 0.5 , suggesting feedback convergence and hence the safe usability of our model. Sample results obtained in our analysis for $\epsilon_{\mathrm{b}}=3.3$ (using both NL and LRA formalisms) and $\epsilon_{\mathrm{b}}=5.45$ (using NL formalism), are presented in Fig. 4.

Observation of all surface plots depicted in Fig. 4 reveals the existence of linewidth variation hot spots (in comparison to the respective far-field linewidths) near the regions where the bare excitonic resonance lies close to the relevant plasmonic resonance $\left(\omega_{0} \approx \omega_{\mathrm{sp}}\right)$. Moreover, it can be seen that these linewidth variations amplify with decreasing $R$ and increasing $N$ which clearly implies contribution of the plasmonically induced fields. We also observe that for some cases (for example, the nonlocally modeled $\epsilon_{\mathrm{b}}=3.3$ case in Fig. 4) the linewidth decreases with decreasing MNP-QE separation $(R)$ for all values of $\omega_{0}$ under study, whereas for other cases (generally, with larger $\max |\beta|$ values), both increasing and decreasing of linewidth against decreasing $R$ were observable in different regions of $\omega_{0}$. To analyze these variations further and gain more insight into the plasmonic contributions, we studied both horizontal and vertical cross sections of the FWHM surface plots, a few samples of which are shown in the fourth and fifth rows of Fig. 4.

As it is observable from the horizontal surface plot cross sections in the the fourth row of Fig. 4, linewidth variation of the $Q_{\mathrm{QE}}$ spectrum (in comparison to the respective far-field value) increases with the number of MNPs $N$ for a given value of $R$ and $\omega_{0}$. That is, irrespective of whether FWHM shows an increasing or a decreasing trend against decreasing $R$, curves with larger values of $N$ show larger linewidth variations [observe how $N=6$ curves are the outermost and $N=2$ curves are the innermost in all three subplots (d), (i), and (n) of Fig. 4]. This is a clear indication of the observed linewidth variation phenomenon being driven by the resultant plasmonic field experienced by the QE.

When we shifted our attention toward the vertical FWHM surface plot cross sections (a few samples of which are depicted in the last row of Fig. 4), we observed a striking resemblance between the shapes of FWHM vs $\hbar \omega_{0}$ plots and the respective $\Omega$ vs $\hbar \omega$ plots. This observation was consistent across the entire parameter range we studied. Compare the shape of Fig. 4(e) against Fig. 5(b) and Fig. 4(o) against Fig. 5(e) to observe this resemblance. It is evident that this observation further validates our earlier claims of plasmonic field influencing the $\mathrm{QE}$ absorption linewidth variations. Furthermore, we observed that the increase or decrease of FWHM with decreasing $R$ (see the sample plots in the fourth row of Fig. 4) was associated with the narrow Fano line shape observed near $\omega \approx \omega_{0}$ in the respective Rabi frequency $\left(\Omega^{r}\right)$ spectra, throughout the parameter region studied. Let us analyze this association using the sample spectra of $\Omega^{r}, \Omega$, and $\eta$ in Fig. 5.

The first row of Fig. 5 depicts the spectra for $\Omega^{r}$ (when $\hbar \omega_{0}=2 \mathrm{eV}$ ), $\Omega$ and $\eta$ for the (nonlocally modeled) case where $\epsilon_{\mathrm{b}}=3.3$. We can readily observe that the absolute values of both $\Omega^{r}$ and $\Omega$ closely align with the respective real-valued components, suggesting a minimal contribution from the respective imaginary parts. Notice again how the shape of $\operatorname{Re}\{\Omega\}$ spectrum strikingly resembles the shape of the FWHM vs $\hbar \omega_{0}$ curve for $\epsilon_{\mathrm{b}}=3.3$ in Fig. 4(e). Let us now recall from our formalism section that $\Omega^{r}=\Omega+\eta(\mathcal{A}-i \mathcal{B})$, extraction of the real part of which yields $\operatorname{Re}\left\{\Omega^{r}\right\}=\operatorname{Re}\{\Omega\}+$ $\operatorname{Re}\{\eta\} \mathcal{A}+\operatorname{Im}\{\eta\} \mathcal{B}$. As $\Omega$ is independent of $\mathrm{QE}$ contributions, the narrow Fano-shaped signature of $\Omega^{r}$ near $\omega \approx \omega_{0}$ forms mainly due to the contributions from $\operatorname{Re}\{\eta\} \mathcal{A}+\operatorname{Im}\{\eta\} \mathcal{B}$ when $\left|\Omega^{r}\right| \approx \operatorname{Re}\left\{\Omega^{r}\right\}$, where $\mathcal{A}$ and $\mathcal{B}$ take the narrow Fano-type line shapes around QE resonance we observed in Fig. 3.

If we vary $\hbar \omega_{0}$ in Fig. 5(a) from $1.5-3 \mathrm{eV}$, the narrow Fano-type line shape now observed around $\hbar \omega \approx 2 \mathrm{eV}$ will traverse from $\approx 1.5-3 \mathrm{eV}$ without a reversal of shape. That is, the Fano-type line shape we observed for $\mathcal{A}$ and $\mathcal{B}$, where a peak is followed by a trough, will be qualitatively retained in $\operatorname{Re}\left\{\Omega^{r}\right\}$ 's Fano signature around QE resonance. However, as $\hbar \omega_{0}$ traverses from $1.5-3 \mathrm{eV}$, the amplitude variation of the 

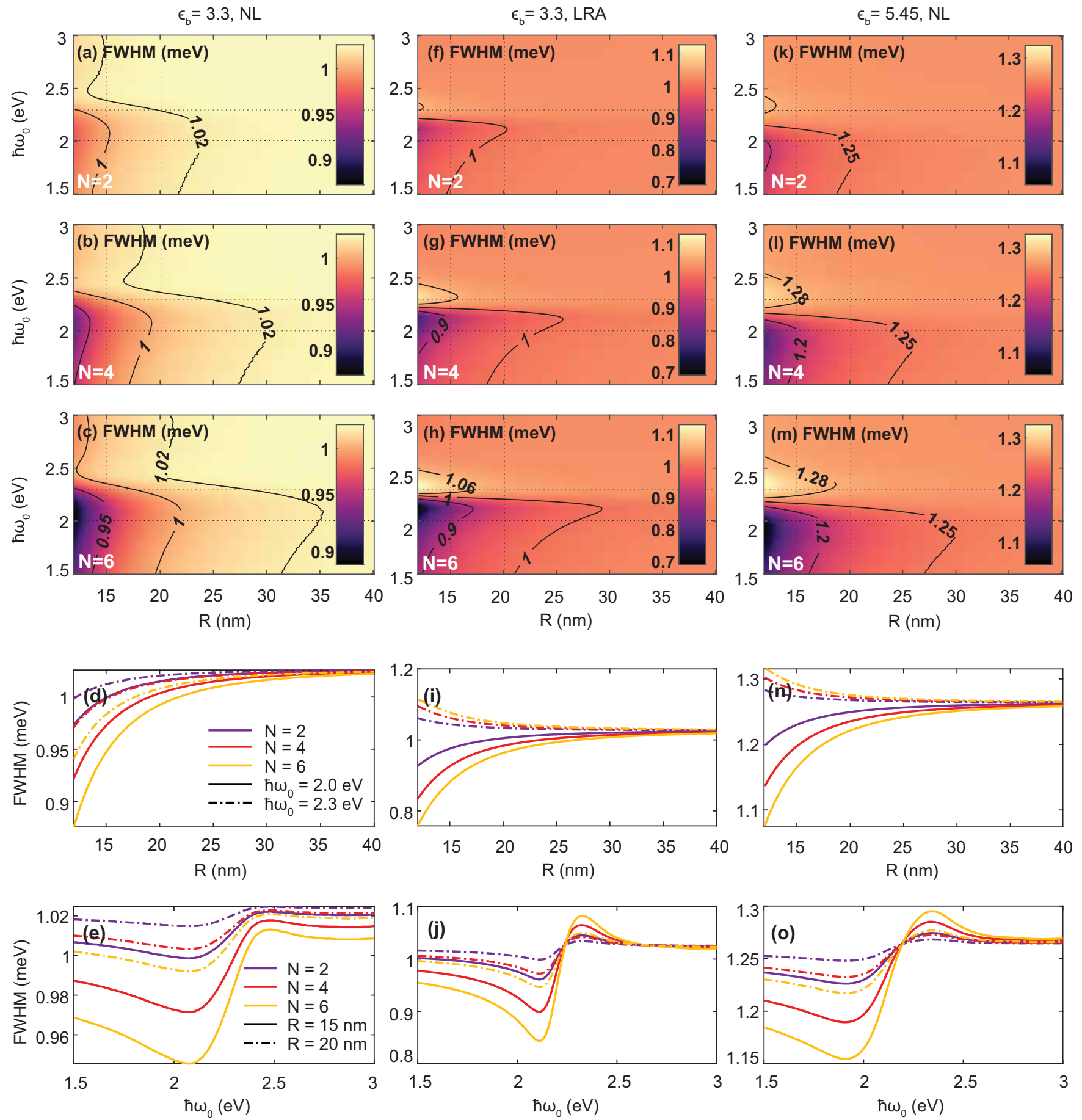

FIG. 4. Variation of full width at half-maximum (FWHM) of the QE absorption rate $\left(Q_{\mathrm{QE}}\right)$ in the presence of $N$ equispaced spherical MNPs. Subplots (a), (b), and (c) of the first column depict the top view of the FWHM surface plots in the presence of $N=2,4$, and 6 MNPs, respectively, plotted against the center separation of each MNP from the QE $(R)$ and the bare excitonic resonance energy $\left(\hbar \omega_{0}\right)$, when the system is submerged in a medium of permittivity $\epsilon_{\mathrm{b}}=3.3$ and the polarizability of each MNP is modeled nonlocally (NL), using the GNOR formalism. (d) Depicts the 2D view of the horizontal cross sections (denoted as black dotted lines at $\hbar \omega_{0}=2 \mathrm{and} 2.3 \mathrm{eV}$ ) in the three preceding FWHM surface plots, where solid lines of respective color represent the cross sections at $\hbar \omega_{0}=2 \mathrm{eV}$ and the dotted-dashed lines represent those at $\hbar \omega=2.3 \mathrm{eV}$, for $N=2$, 4, and 6. Similarly, (e) depicts the vertical cross sections shown as dashed lines in the FWHM surface plots (a), (b), and (c), where the solid lines represent the cross sections at $R=15 \mathrm{~nm}$ and the dotted-dashed lines represent those at $R=20 \mathrm{~nm}$. The second and third columns represent similar arrangements of plots for cases where $\epsilon_{\mathrm{b}}=3.3$ with the MNP polarizability modeled using the local response approximation (LRA), and for $\epsilon_{\mathrm{b}}=5.45$ modeled using the nonlocal GNOR formalism, respectively. The legends shown in (d) and (e) are common to all plots in the row. 

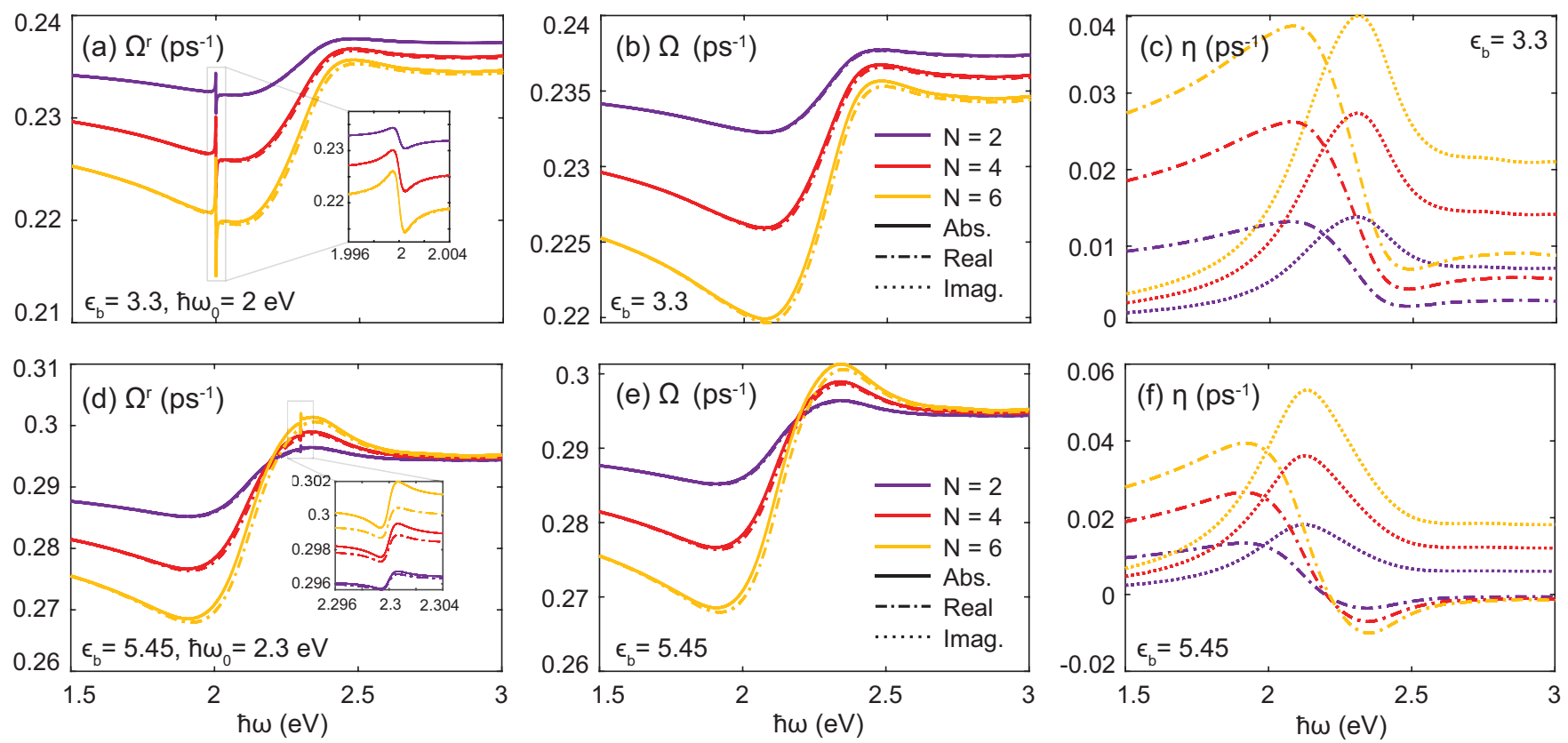

FIG. 5. (a) Effective or normalized Rabi frequency $\Omega^{r}$ experienced by a quantum emitter with bare excitonic energy $\hbar \omega_{0}=2 \mathrm{eV}$ under the influence of $N=2,4$, and 6 equispaced MNPs, each located at a distance $R=15 \mathrm{~nm}$ from the QE, when the entire system is submerged in a medium of relative permittivity $\epsilon_{\mathrm{b}}=3.3$. (b) The corresponding Rabi frequency in the absence of coherences $(\Omega)$. (c) The coefficient of QE self-interaction $(\eta)$. The legend in the middle subplot is common to the entire row where the solid, dotted-dashed, and dotted lines correspond to the relevant absolute, real, and imaginary quantities, respectively. The second row depicts an arrangement of plots similar to the earlier, for the parameters $\epsilon_{\mathrm{b}}=5.45, \hbar \omega_{0}=2.3 \mathrm{eV}$, and $R=15 \mathrm{~nm}$. Insets in (a) and (d) are enlargements along the frequency axis around $\hbar \omega_{0}$, for better visibility of the spectral signatures.

Fano peak and trough will be governed by $\operatorname{Re}\{\eta\}$ and $\operatorname{Im}\{\eta\}$ indicated in Fig. 5(c). When both $\operatorname{Re}\{\eta\}$ and $\operatorname{Im}\{\eta\}$ take large positive values, the similarly shaped Fano patterns of $\mathcal{A}$ and $\mathcal{B}$ will be linearly (additively) combined to give the enhanced Fano pattern we observe for $\operatorname{Re}\left\{\Omega^{r}\right\}=\operatorname{Re}\{\Omega\}+\operatorname{Re}\{\eta\} \mathcal{A}+$ $\operatorname{Im}\{\eta\} \mathcal{B}$ around $\omega \approx \omega_{0}$ when $\hbar \omega_{0}=2 \mathrm{eV}$ in Fig. 5(a). When $\hbar \omega_{0} \rightarrow 1.5 \mathrm{eV}$ or $3 \mathrm{eV}$ (the two extreme points along the $x$ axis), the peak and trough amplitudes of $\operatorname{Re}\left\{\Omega^{r}\right\}$ 's narrow Fano pattern will comparatively reduce due to the diminishing but positive $\operatorname{Re}\{\eta\}$ and $\operatorname{Im}\{\eta\}$ values observed in Fig. 5(c) at the two extreme ends along the $x$ axis. Notice how the Fano signature of $\operatorname{Re}\left\{\Omega^{r}\right\}$ enhances with increasing $N$ due to increased plasmonic impact, which is also explainable by the aforementioned formula for $\operatorname{Re}\left\{\Omega^{r}\right\}$. In essence, when both $\operatorname{Re}\{\eta\}$ and $\operatorname{Im}\{\eta\}$ are positive, the Fano signature of $\operatorname{Re}\left\{\Omega^{r}\right\}$ will not reverse in shape along the $x$ axis, but its magnitude will be governed by $\operatorname{Re}\{\eta\}$ and $\operatorname{Im}\{\eta\}$.

If we now shift our attention to Fig. 5(d) which depicts the variation of real and absolute spectra of $\Omega^{r}$ at $\epsilon_{\mathrm{b}}=5.45$ and $\hbar \omega_{0}=2.3 \mathrm{eV}$, we can observe a reversed and diminished Fano signature around $\hbar \omega_{0}$ in comparison to our earlier observation in Fig. 5(a). This variation too can be explained using our earlier formula $\operatorname{Re}\left\{\Omega^{r}\right\}=\operatorname{Re}\{\Omega\}+\operatorname{Re}\{\eta\} \mathcal{A}+\operatorname{Im}\{\eta\} \mathcal{B}$ as follows. Comparison of Fig. 5(d) against Fig. 5(f) reveals that $\operatorname{Re}\{\eta\}$ is now negative near $\hbar \omega_{0}=2.3 \mathrm{eV}$. Such negation of $\operatorname{Re}\{\eta\}$ is highly likely to reverse the Fano line shape resulting from the $\operatorname{Re}\{\eta\} \mathcal{A}+\operatorname{Im}\{\eta\} \mathcal{B}$ component of $\operatorname{Re}\left\{\Omega^{r}\right\}$ as $\mathcal{A}$ is usually sufficiently larger in magnitude than $\mathcal{B}$ (for example, see Fig. 3). The diminishing of Fano amplitude results from the competition between the now differently signed $\mathcal{A}$ and $\mathcal{B}$.
For all our observations in the wide parameter region considered (for both LRA and GNOR based models), the MNP-QE constellations exhibiting patterns of increasing FWHM against decreasing $R$ [for example, the dotted dashed lines in Figs. 4(i) and 4(n)] could be mapped to regions of negative $\operatorname{Re}\{\eta\}$, and hence reversed Fano shapes of $\operatorname{Re}\left\{\Omega^{r}\right\}$ near the respective excitonic resonance $\hbar \omega_{0}$, as explained above. On the contrary, the cases exhibiting patterns of decreasing FWHM against decreasing $R$ [for example, all curves in Fig. 4(d) and the solid lines in Figs. 4(i) and 4(n)] could be mapped to regions of positive $\operatorname{Re}\{\eta\}$, and hence nonreversed Fano shapes of $\operatorname{Re}\left\{\Omega^{r}\right\}$ near $\hbar \omega_{0}$.

\section{Coupled-plasmon-induced excitonic energy and dephasing rate shifts}

Let us analyze the influence of the weakly intercoupled plasmonic ring of equispaced spherical MNPs on the excitonic energy and the dephasing rate of the $\mathrm{QE}$ at the center. In the first and second rows of Fig. 6, we have shown the spectra of QE exciton transition energy redshift $\left(\Pi_{f}=\operatorname{Re}\{\eta\} \Delta\right)$ and QE dephasing rate blueshift $\left(\Lambda_{f}=\operatorname{Im}\{\eta\} \Delta\right)$ due to the surrounding $N=1, \ldots, 6 \mathrm{MNPs}$, for three sample cases where $\epsilon_{\mathrm{b}}=$ $3.3, \hbar \omega_{0}=2 \mathrm{eV}$ (first column), $\epsilon_{\mathrm{b}}=5.45, \hbar \omega_{0}=2 \mathrm{eV}$ (second column), and $\epsilon_{\mathrm{b}}=5.45, \hbar \omega_{0}=2.3 \mathrm{eV}$ (third column). As is also suggested by their equations, $\Pi_{f}$ and $\Lambda_{f}$ exactly follow the variations of $\operatorname{Re}\{\eta\}$ and $\operatorname{Im}\{\eta\}$, respectively, except at the sharp narrow slits toward zero around QE resonance, resulting from the spectrum of $\Delta$ (which goes to zero near QE resonance and to 1 as the external field detunes from 

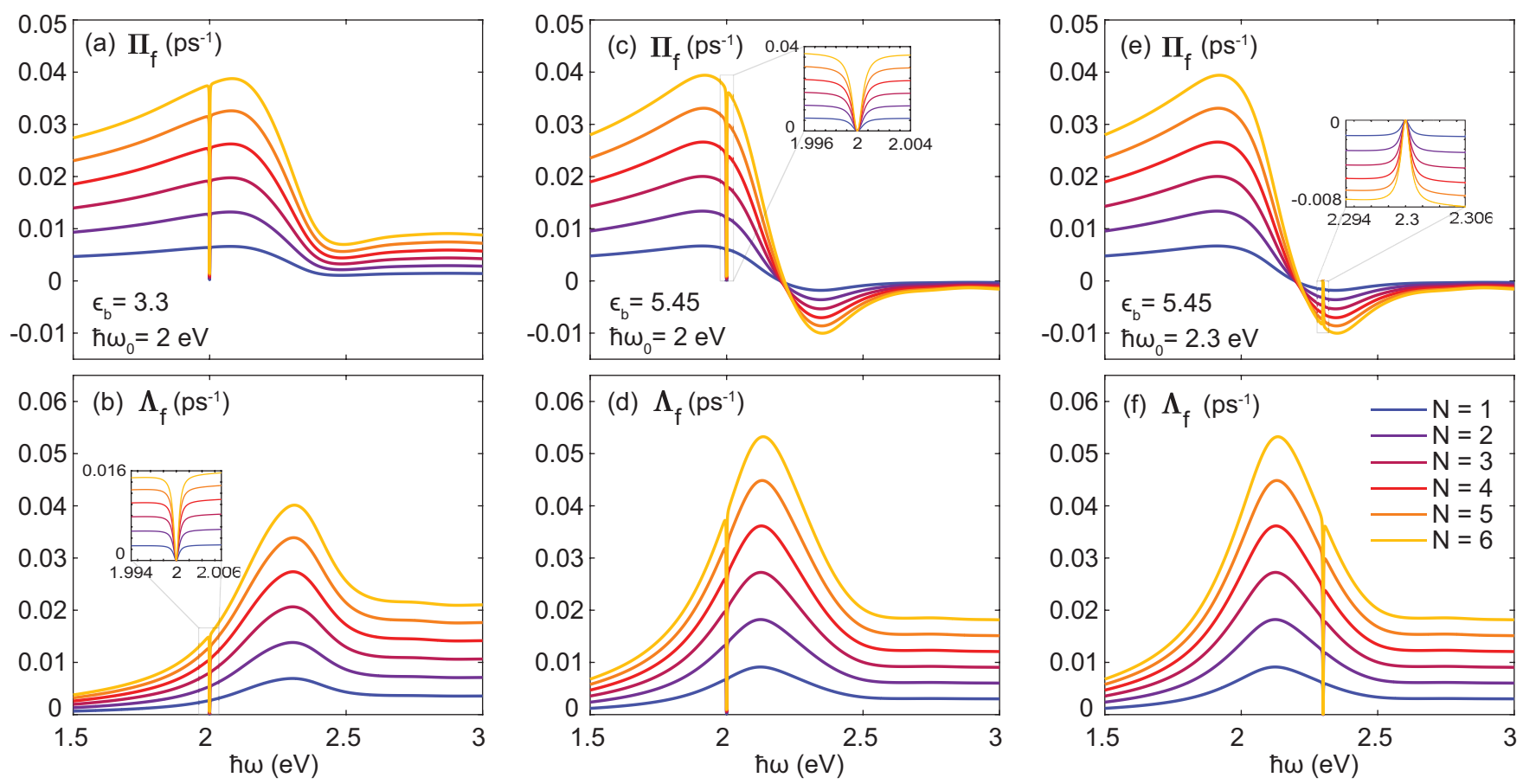

FIG. 6. (a) The exciton transition energy redshift $\left(\Pi_{f}\right)$ experienced by a quantum emitter with bare excitonic energy $\hbar \omega_{0}=2 \mathrm{eV}$ surrounded by $N=1,2, \ldots, 6 \mathrm{MNPs}$, each located at a distance $R=15 \mathrm{~nm}$ and submerged in a medium of relative permittivity $\epsilon_{\mathrm{b}}=3.3$. (b) Exciton dephasing rate blueshift $\left(\Lambda_{f}\right)$ experienced by the same QE. The second and third columns show similar constellations of plots for $\epsilon_{\mathrm{b}}=5.45, \hbar \omega_{0}=2 \mathrm{eV}$ and $\epsilon_{\mathrm{b}}=5.45, \hbar \omega_{0}=2.3 \mathrm{eV}$, respectively. The insets in (b), (c), and (e) are enlargements along the frequency axis around $\hbar \omega_{0}$, for better visibility of the spectral signatures.

the QE resonance, as we saw in Fig. 3). As $\operatorname{Im}\{\eta\}>0$ for all three cases observed, the $\mathrm{QE}$ experiences dephasing rate blueshifts in the entire range where $\hbar \omega$ varies from 1.5 to $3 \mathrm{eV}$, except when $\omega=\omega_{0}$ where $\Lambda_{f}=0$, for all values of $N$. The observed dephasing rate blueshift is seen to increase with $N$, throughout the spectral region studied.

It is evident that the $\mathrm{QE}$ experiences plasmon-induced transition energy redshifts in the regions where $\operatorname{Re}\{\eta\}>0$ and blueshifts where $\operatorname{Re}\{\eta\}<0$ (except at $\omega=\omega_{0}$ ). Thus, our results suggest that, for QEs under the influence of weakly intercoupled symmetric planar MNP systems, parameter regions exhibiting trends of decreasing $Q_{\mathrm{QE}}$ linewidths against decreasing MNP-QE center separations $R$ are likely to be associated with plasmon-induced excitonic energy redshifts [for example, compare Fig. 4(d) with Fig. 6(a)], as both these phenomena occur when $\operatorname{Re}\{\eta\}>0$. Similarly, regions exhibiting trends of increasing $Q_{\mathrm{QE}}$ linewidths against decreasing MNP-QE center separations $R$ are likely to be associated with plasmon-induced excitonic energy blueshifts [compare the dotted-dashed lines in Fig. 4(n) with Fig. 6(e)], as both these phenomena occur when $\operatorname{Re}\{\eta\}<0$. Moreover, irrespective of the shift type (redshift/blueshift), the magnitude of the plasmon-induced excitonic energy shift tends to increase with $N$.

\section{Collective absorption}

Even though the main objective of this paper is to analyze the influence of a planar metal nanoparticle assembly on the optical response of a quantum emitter, we briefly discuss the absorption properties of the entire system in this section.
Figure 7 depicts the estimated absorption rate spectra $\left(Q_{\text {tot }}\right)$ for symmetric planar MNP-QE nanohybrids immersed in an aqueous medium $\left(\epsilon_{\mathrm{b}}=1.78\right)$, separate $\mathrm{MNP}\left(N Q_{\mathrm{n}}\right)$ and $\mathrm{QE}$ $\left(Q_{\mathrm{QE}}\right)$ contributions to the aforementioned spectra, as well as the collective MNP spectra $\left(Q_{\mathrm{n}_{-} w c}\right)$ in the absence of the $\mathrm{QE}$ (or coherences). The juxtaposition of $Q_{\mathrm{tot}}$ against $N Q_{\mathrm{n}}$ reveals that the combined spectrum of the entire planar nanohybrid is largely dominated by the MNP absorption component, whereas the contribution of the QE is minute when considered solely in the context of magnitude. However, a comparison between the collective MNP absorption spectra in the presence and absence of the QE (or coherences) reveals that the large Fano-type interference pattern in the MNP spectra (and hence in the collective MNP-QE nanohybrid spectra) is entirely attributable to the alteration of the resultant field caused by the sharp QE response. This phenomenon of dramatic modification of the nanohybrid spectrum by a small $\mathrm{QE}$ spectrum is similarly observed for a single MNP-QE nanohybrid in the context of scattering [28].

\section{E. Practical considerations and potential applications}

The quantum emitter considered here for the MNP-QE constellation was a generic two-level system of negligible radius embedded in a homogeneous effective medium (material) with relative permittivity $\epsilon_{\mathrm{s}}$. In a real-world system, the aforementioned emitter can be a small quantum dot or a photoactive molecule. Optical properties of semiconductor quantum dots are usually described using homogeneous effective medium approximation, which is applicable as long as the optical wavelength in the structure is much larger 

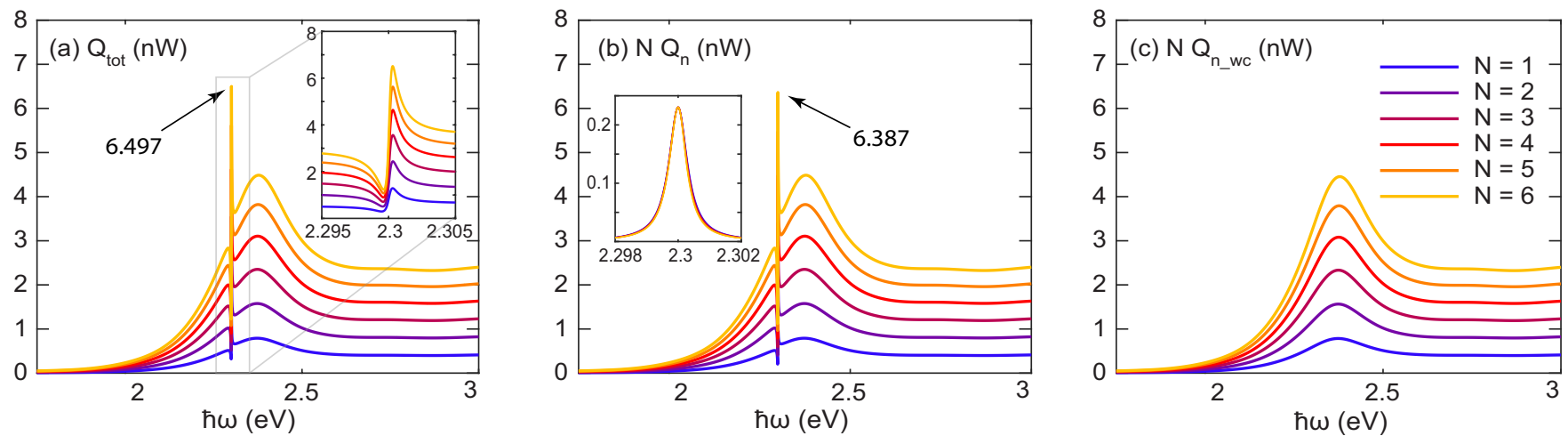

FIG. 7. (a) Estimated average absorption rate $\left(Q_{\text {tot }}\right)$ for a symmetric planar MNP-QE setup comprising $N=1,2, \ldots, 6$ equidistant MNPs immersed in an aqueous medium $\left(\epsilon_{\mathrm{b}} \approx 1.78\right)$, each located at a distance of $15 \mathrm{~nm}$ from a QE of excitonic energy $\hbar \omega_{0}=2.3 \mathrm{eV}$. The inset is an enlargement along the $x$ axis near $\hbar \omega \approx 2.3 \mathrm{eV}$. (b) Collective absorption rate spectra of $N=1,2, \ldots, 6$ MNPs under the influence of the $\mathrm{QE}$ and the external field. The inset shows the corresponding QE spectra. (c) Collective absorption rate spectra of $N=1,2, \ldots, 6 \mathrm{MNPs}$ in the absence of the QE (or without coherences). The legend is common to all three subplots.

than the relevant length scales of the system [56]. However, the nonlocal character of the relation between the electric field and the polarization of the QD comes into play near sharp excitonic resonances when the QD becomes comparable with the exciton Bohr radius [56,57]. Fluorescent molecules could also possess nonlocal optical polarizabilities of the form $\alpha\left(\boldsymbol{r}, \boldsymbol{r}^{\prime}, \omega\right)$, which relates the polarization at each point $\boldsymbol{r}$ in the molecule to the electric field at all $\boldsymbol{r}^{\prime}$ points in the molecule $E\left(\boldsymbol{r}^{\prime}\right)$ [58]. Thus, a possible future extension of the presented generic formalism could be incorporation of emitter nonlocal effects for specific types of molecular emitters.

As a concluding remark, we recall that the unique optics of QEs related to absorption and fluorescence can be tailored for a broad range of potential applications such as plasmonic lasers $[14,15,59]$, quantum information processors [2], various in vivo nanodevices such as sensors and switches $[5,11,60]$, and photovoltaic cells [61] using the near fields of plasmonic nanoparticles. Our fully analytical model capable of accommodating multiple MNPs enables easy optimization of the large system parameter space for such applications. This is vital for the advancement of the aforementioned technologies toward the level of device realization [15].

\section{CONCLUSION}

We developed an analytical framework to study the influence of a weakly intercoupled in-plane spherical metal nanoparticle (MNP) assembly on a coherently illuminated quantum emitter (QE), using the generalized nonlocal optical response (GNOR) theory. We reduced the derived equations into simple and concise expressions representing a QE mediated by a symmetric MNP setup. Using the derived model, we analyzed the optical properties of a coherently illuminated $\mathrm{QE}$ at the center of an in-plane symmetric MNP setup. We observed that the QE experiences plasmon-induced absorption rate spectral linewidth variations that increase in magnitude with decreasing MNP-QE center separation and increasing number of MNPs. We could also observe that the parameter regions where the $\mathrm{QE}$ exhibits trends of decreasing linewidth against decreasing MNP-QE center separation are likely to be associated with plasmon-induced excitonic energy redshifts, and vice versa. The magnitude of the observed exciton energy redshift/blueshift was seen to increase with the number of MNPs in the constellation. We also demonstrated the ability of the QE to dramatically modify the collective absorption rate spectrum of the entire nanohybrid, even when the magnitude of the QE spectrum is considerably smaller than that of the isolated MNPs.

\section{ACKNOWLEDGMENTS}

H.H. gratefully acknowledges P. Mulvaney, A. M. Funston, and D. Kudavithana for their support and fruitful discussions. This research was funded by the Australian Research Council under Grant No. CE170100026.

\section{APPENDIX A: EXTERNAL FIELD FEEDBACK DIPOLES}

The 0th level external field feedback dipole, or the dipole moment component formed directly due to the external field (with a positive-frequency component $E^{+}=E_{0} e^{-i \omega t}$ ), in an $f_{0}$ th MNP can be found using (2) as

$$
d_{f_{0}}^{+}=\left(4 \pi \epsilon_{0} \epsilon_{\mathrm{b}}\right) r_{f_{0}}^{3} \beta_{f_{0}} E_{0} e^{-i \omega t} .
$$

Collective first-level feedback dipole induced in the nth MNP due to all such 0 th level dipoles (where the dipole index $f_{0} \neq$ n) can be obtained with the aid of (5) as

$$
\begin{aligned}
d_{\mathrm{n}_{-} \Sigma f_{0}}^{+} & =\left(4 \pi \epsilon_{0} \epsilon_{\mathrm{b}}\right) r_{\mathrm{n}}^{3} \beta_{\mathrm{n}} s_{\alpha} E_{0} e^{-i \omega t} \sum_{\substack{f_{0}=1 \\
f_{0} \neq \mathrm{n}}}^{N}\left(\frac{r_{f_{0}}^{3} \beta_{f_{0}}}{R_{\mathrm{n} f_{0}}^{3}}\right) \\
& =d_{\mathrm{n}}^{+} s_{\alpha} \sum_{\substack{f_{0}=1 \\
f_{0} \neq \mathrm{n}}}^{N}\left(\frac{r_{f_{0}}^{3} \beta_{f_{0}}}{R_{\mathrm{n} f_{0}}^{3}}\right) .
\end{aligned}
$$

Similarly, using the first-level feedback pattern above, the second-level external-field feedback dipole induced in the nth MNP due to the collective first-level external-field feedback dipoles induced in all surrounding MNPs (indexed in the sum- 
mation using the subscript $f_{1}$, where $f_{1} \neq n$ ) can be obtained as

$$
d_{\mathrm{n}_{-} \Sigma f_{1} \_\Sigma f_{0}}^{+}=d_{\mathrm{n}}^{+} s_{\alpha}^{2} \sum_{\substack{f_{1}=1 \\ f_{1} \neq \mathrm{n}}}^{N}\left(\frac{r_{f_{1}}^{3} \beta_{f_{1}}}{R_{\mathrm{n} f_{1}}^{3}} \sum_{\substack{f_{0}=1 \\ f_{0} \neq f_{1}}}^{N} \frac{r_{f_{0}}^{3} \beta_{f_{0}}}{R_{f_{1} f_{0}}^{3}}\right) .
$$

By repeating the above procedure, the pth-level feedback dipole formed in the nth MNP due to the collective $(\mathrm{p}-1)$ th level dipoles in the surrounding MNPs (indexed in the summation using the subscript $f_{\mathrm{p}-1}$ ) can be found, which results in Eq. (10) in the main text.

\section{APPENDIX B: ESTIMATING THE MNP ABSORPTION RATE}

In this Appendix, we extend the procedure followed in literature $[2,53]$ to obtain the MNP absorption rate for a single MNP-QE nanohybrid toward our symmetric multiple MNP constellation.

The total dipole moment induced in the jth MNP in a symmetric constellation can be obtained using (30) by substituting $\mathrm{j}$ in place of $\mathrm{n}$. The total field incident on the nth MNP comprises the sum of the dipole response fields of all such jth MNPs (where $\mathrm{j} \neq \mathrm{n}$ ), transition dipole response field of the $\mathrm{QE}$, and the externally illuminated field. Its positive-frequency component can be computed as

$$
E_{\mathrm{n} \_ \text {out }}^{+}=\sum_{\substack{\mathrm{j}=1 \\ \mathrm{j} \neq \mathrm{n}}}^{N} \frac{s_{\alpha}\left[d_{\mathrm{j} \_ \text {tot }}^{+}\right]_{\mathrm{sym}}}{\left(4 \pi \epsilon_{0} \epsilon_{\mathrm{b}}\right) R_{\mathrm{nj}}^{3}}+\frac{s_{\alpha} d_{\mathrm{QE}}^{+}}{\left(4 \pi \epsilon_{0} \epsilon_{\mathrm{b}}\right) R^{3} \epsilon_{\mathrm{effS}}}+E_{0} e^{-i \omega t} .
$$

Positive-frequency component of the field inside the $n$th MNP can be found by accounting for the screening of the incident field by the MNP dielectric, in the same way we accounted for the screening of the QE material as follows $[2,53]$ :

$$
E_{\mathrm{n} \_ \text {in }}^{+}=\frac{E_{\mathrm{n} \_ \text {out }}^{+}}{\epsilon_{\mathrm{effM}}} \text {, where } \epsilon_{\mathrm{effM}}=\frac{2 \epsilon_{\mathrm{b}}+\epsilon_{\mathrm{m}}}{3 \epsilon_{\mathrm{b}}} .
$$

It is worth noticing that the total dipole moment of the jth MNP obtained using Eq. (30) embeds the nonlocally modeled Clausius Mossotti factor $\beta$, whereas the MNP screening factor $\epsilon_{\mathrm{effM}}$ contains the local MNP permittivity $\epsilon_{\mathrm{m}}$ outside the aforementioned $\beta$ where nonlocality has been accounted for. It is therefore important to avoid this local-nonlocal incompatibility by estimating the MNP absorption rate using LRA by setting $\delta_{\mathrm{NL}} \rightarrow 0$ and hence $\beta \rightarrow \beta_{\text {LRA }}$ only when using the MNP (or collective hybrid molecule) absorption rate equation(s) derived in this paper.

We simplify (B2) using $d_{\mathrm{QE}}^{+}=\mu \tilde{\rho}_{\mathrm{eg}} e^{-i \omega t}$, (9), (14), and (30) to obtain

$$
E_{\mathrm{n} \_ \text {in }}=\frac{\tilde{E}_{\mathrm{n} \_ \text {out }}^{+} e^{-i \omega t}}{\epsilon_{\mathrm{effM}}}+\frac{\tilde{E}_{\mathrm{n} \_ \text {out }}^{-} e^{+i \omega t}}{\epsilon_{\mathrm{effM}}^{*}},
$$

where $\tilde{E}_{\text {n_out }}^{-}=\left(\tilde{E}_{\text {n_out }}^{+}\right)^{*}$ and

$$
\tilde{E}_{\mathrm{n} \_ \text {out }}^{+}=\left(E_{0}+\frac{s_{\alpha} \mu \tilde{\rho}_{\mathrm{eg}}}{\left(4 \pi \epsilon_{0} \epsilon_{\mathrm{b}}\right) R^{3} \epsilon_{\mathrm{effS}}}\right) \frac{1}{\left(1-s_{\alpha} r^{3} \beta_{\mathrm{LRA}} \zeta\right)} .
$$

We now obtain the polarization current density of the nth MNP in our constellation as the time derivative of its polarization (dipole moment per unit volume) [2,53] as follows:

$$
j=\frac{\partial}{\partial t}\left\{\frac{\left[d_{\mathrm{n} \_ \text {tot }}^{+}\right]_{\mathrm{sym}}+\text { c.c. }}{V}\right\}
$$

where $V=(4 / 3) \pi r^{3}$ is the volume of the nth MNP. This can be simplified using (9), (14), and (30) to obtain

$$
j=\tilde{j}^{+} e^{-i \omega t}+\tilde{j}^{-} e^{+i \omega t},
$$

where $\tilde{j}^{-}=\left(\tilde{j}^{+}\right)^{*}$ and

$$
\tilde{j}^{+}=\left[E_{0}+\frac{s_{\alpha} \mu \tilde{\rho}_{\mathrm{eg}}}{4 \pi \epsilon_{0} \epsilon_{\mathrm{b}} \epsilon_{\mathrm{effS}} R^{3}}\right] \frac{4 \pi \epsilon_{0} \epsilon_{\mathrm{b}} r^{3}(-i \omega) \beta_{\mathrm{LRA}}}{V\left(1-s_{\alpha} r^{3} \beta_{\mathrm{LRA}} \zeta\right)} .
$$

Now, the average energy absorption rate of the $n$th MNP can be obtained using the volume integral $[2,53]$

$$
Q_{\mathrm{n}}=\left\langle Q_{\mathrm{n}}(t)\right\rangle=\left\langle\int j E_{\mathrm{n} \_ \text {in }} d v\right\rangle,
$$

where $\langle\ldots\rangle$ is the time average defined as

$$
\langle f(t)\rangle=(\omega / 2 \pi) \int_{0}^{2 \pi / \omega} f(t) d t .
$$

It is evident that $Q_{\mathrm{n}}$ takes the form

$$
Q_{\mathrm{n}}=\left[\frac{\tilde{j}^{-} \tilde{E}_{\mathrm{n} \_ \text {out }}^{+}}{\epsilon_{\mathrm{effM}}}+\frac{\tilde{j}^{+} \tilde{E}_{\mathrm{n} \_ \text {out }}^{-}}{\epsilon_{\mathrm{effM}}^{*}}\right] V,
$$

which can be further simplified to arrive at Eq. (46) in the main text. We can obtain the MNP absorption rate equation for the single MNP-QE nanohybrid already available in literature [2,53] by setting $\zeta=0$ in $Q_{\mathrm{n}}$ :

$$
\begin{aligned}
\left.Q_{\mathrm{MNP}}\right|_{N=1} & =2 \pi \epsilon_{0} \epsilon_{\mathrm{b}} r^{3} \omega\left(E_{\mathrm{c}}^{2}+E_{\mathrm{s}}^{2}\right) \operatorname{Im}\left\{\frac{\beta_{\mathrm{LRA}}}{\epsilon_{\mathrm{effM}}^{*}}\right\}, \\
E_{\mathrm{c}} & =2 E_{0}+\frac{s_{\alpha} \mu \mathcal{A}}{\left(2 \pi \epsilon_{0} \epsilon_{\mathrm{b}}\right) \epsilon_{\mathrm{effS}} R^{3}}, \\
E_{\mathrm{s}} & =\frac{s_{\alpha} \mu \mathcal{B}}{\left(2 \pi \epsilon_{0} \epsilon_{\mathrm{b}}\right) \epsilon_{\mathrm{effS}} R^{3}},
\end{aligned}
$$

where $2 E_{0}$ should be replaced by $E_{0}$ where the incident field takes the form $E=E_{0} \cos (\omega t)$. 
[1] H. P. Hapuarachchi, Analysis of exciton-plasmon nanohybrids, Ph.D. thesis, Monash University, Clayton, Australia, 2019, https://doi.org/10.26180/5cfdcd6c18b1e.

[2] R. D. Artuso, The optical response of strongly coupled quantum dot-metal nanoparticle hybrid systems, Ph.D. thesis, University of Maryland, College Park, Maryland, 2012, https://drum.lib. umd.edu/handle/1903/13644.

[3] S. A. Maier, Plasmonics: Fundamentals and Applications (Springer, New York, 2007), Chap. 5.

[4] M. Premaratne and G. P. Agrawal, Light Propagation in Gain Media: Optical Amplifiers (Cambridge University Press, Cambridge, 2011).

[5] H. Hapuarachchi and M. Premaratne, in 2018 IEEE 12th International Conference on Nano/Molecular Medicine and Engineering (NANOMED) (IEEE, Piscataway, NJ, 2018), pp. 20-24.

[6] V. Senevirathne, H. Hapuarachchi, S. Mallawaarachchi, S. D. Gunapala, M. I. Stockman, and M. Premaratne, J. Phys.: Condens. Matter 31, 085302 (2019).

[7] K. Gettapola, H. Hapuarachchi, M. I. Stockman, and M. Premaratne, J. Phys.: Condens. Matter 32, 125301 (2019).

[8] M. Sukharev and A. Nitzan, J. Phys.: Condens. Matter 29, 443003 (2017).

[9] J. N. Anker, W. P. Hall, O. Lyandres, N. C. Shah, J. Zhao, and R. P. Van Duyne, Nanoscience and Technology: A Collection of Reviews from Nature Journals (World Scientific, Singapore, 2010), pp. 308-319.

[10] H. Zhou, J. Liu, and S. Zhang, TrAC, Trends Anal. Chem. 67, 56 (2015).

[11] H. Hapuarachchi, S. D. Gunapala, and M. Premaratne, J. Phys.: Condens. Matter 31, 325301 (2019).

[12] S. Mallawaarachchi, M. Premaratne, and P. K. Maini, IEEE J. Sel. Top. Quantum Electron. 25, 1 (2018).

[13] C. Jayasekara, M. Premaratne, M. I. Stockman, and S. D. Gunapala, J. Appl. Phys. 118, 173101 (2015).

[14] D. Lelwala Gamacharige, S. D. Gunapala, M. I. Stockman, and M. Premaratne, Phys. Rev. B 99, 115405 (2019).

[15] L. Kumarapperuma, M. Premaratne, P. K. Jha, M. I. Stockman, and G. P. Agrawal, Appl. Phys. Lett. 112, 201108 (2018).

[16] D. Weeraddana, M. Premaratne, S. D. Gunapala, and D. L. Andrews, Phys. Rev. B 94, 085133 (2016).

[17] C. Abeywickrama, M. Premaratne, S. D. Gunapala, and D. L. Andrews, J. Phys.: Condens. Matter 32, 095305 (2019).

[18] R. T. Wijesekara, S. D. Gunapala, M. I. Stockman, and M. Premaratne, Phys. Rev. B 101, 245402 (2020).

[19] J. Tang and E. H. Sargent, Adv. Mater. 23, 12 (2011).

[20] Q. Lin, A. Armin, R. C. R. Nagiri, P. L. Burn, and P. Meredith, Nat. Photonics 9, 106 (2015).

[21] S. Sadeghi, Nanotechnology 21, 355501 (2010).

[22] A. O. Govorov, G. W. Bryant, W. Zhang, T. Skeini, J. Lee, N. A. Kotov, J. M. Slocik, and R. R. Naik, Nano Lett. 6, 984 (2006).

[23] T. Hartsfield, W.-S. Chang, S.-C. Yang, T. Ma, J. Shi, L. Sun, G. Shvets, S. Link, and X. Li, Proc. Natl. Acad. Sci. U.S.A. 112, 12288 (2015).

[24] H. Zhang, M. Li, K. Wang, Y. Tian, J.-S. Chen, K. T. Fountaine, D. DiMarzio, M. Liu, M. Cotlet, and O. Gang, ACS Nano 14, 1369 (2019).

[25] F. Nicoli, T. Zhang, K. Hübner, B. Jin, F. Selbach, G. Acuna, C. Argyropoulos, T. Liedl, and M. Pilo-Pais, Small 15, 1804418 (2019).
[26] T. Warnakula, S. D. Gunapala, M. I. Stockman, and M. Premaratne, Phys. Rev. B 100, 085439 (2019).

[27] A. Devi, S. D. Gunapala, M. I. Stockman, and M. Premaratne, Phys. Rev. A 102, 013701 (2020).

[28] H. Hapuarachchi, S. Mallawaarachchi, H. T. Hattori, W. Zhu, and M. Premaratne, J. Phys.: Condens. Matter 30, 054006 (2018).

[29] Y. Liu, X. Dai, S. Mallawaarachchi, H. Hapuarachchi, Q. Shi, D. Dong, S. H. Thang, M. Premaratne, and W. Cheng, J. Mater. Chem. C 5, 10926 (2017).

[30] H. Hapuarachchi, M. Premaratne, Q. Bao, W. Cheng, S. D. Gunapala, and G. P. Agrawal, Phys. Rev. B 95, 245419 (2017).

[31] A. Ridolfo, O. Di Stefano, N. Fina, R. Saija, and S. Savasta, Phys. Rev. Lett. 105, 263601 (2010).

[32] H. Hapuarachchi, S. D. Gunapala, Q. Bao, M. I. Stockman, and M. Premaratne, Phys. Rev. B 98, 115430 (2018).

[33] A. Hatef, S. Sadeghi, and M. R. Singh, Nanotechnology 23, 205203 (2012).

[34] S. Raza, S. I. Bozhevolnyi, M. Wubs, and N. A. Mortensen, J. Phys.: Condens. Matter 27, 183204 (2015).

[35] R. D. Artuso and G. W. Bryant, Nano Lett. 8, 2106 (2008).

[36] N. A. Mortensen, S. Raza, M. Wubs, T. Søndergaard, and S. I. Bozhevolnyi, Nat. Commun. 5, 3809 (2014).

[37] J. Tiggesbäumker, L. Köller, K.-H. Meiwes-Broer, and A. Liebsch, Phys. Rev. A 48, R1749 (1993).

[38] M. Wubs and N. A. Mortensen, Quantum Plasmonics (Springer, Berlin, 2017), pp. 279-302.

[39] S. Raza, N. Stenger, S. Kadkhodazadeh, S. V. Fischer, N. Kostesha, A.-P. Jauho, A. Burrows, M. Wubs, and N. A. Mortensen, Nanophotonics 2, 131 (2013).

[40] S. Raza, W. Yan, N. Stenger, M. Wubs, and N. A. Mortensen, Opt. Express 21, 27344 (2013).

[41] J. Zuloaga, E. Prodan, and P. Nordlander, Nano Lett. 9, 887 (2009).

[42] K. Andersen, K. W. Jacobsen, and K. S. Thygesen, Phys. Rev. B 86, 245129 (2012).

[43] J. E. Moore, Describing the electronic structure of molecules on metal surfaces, The Pennsylvania State University, 2015, https: //etda.libraries.psu.edu/catalog/27120.

[44] S. Mallawaarachchi, M. Premaratne, S. D. Gunapala, and P. K. Maini, Phys. Rev. B 95, 155443 (2017).

[45] W. Zhu, R. Esteban, A. G. Borisov, J. J. Baumberg, P. Nordlander, H. J. Lezec, J. Aizpurua, and K. B. Crozier, Nat. Commun. 7, 11495 (2016).

[46] H. Wang, K. Chen, J. Pan, S. Huang, J. Lin, W. Xie, and X. Huang, J. Quant. Spectrosc. Radiat. Transfer 245, 106878 (2020).

[47] P. Nordlander, C. Oubre, E. Prodan, K. Li, and M. Stockman, Nano Lett. 4, 899 (2004).

[48] K.-H. Su, Q.-H. Wei, X. Zhang, J. Mock, D. R. Smith, and S. Schultz, Nano Lett. 3, 1087 (2003).

[49] D. J. Griffiths and R. College, Introduction to Electrodynamics, Vol. 3 (Prentice Hall, Upper Saddle River, NJ, 1999), Chap. 3.

[50] A. Yariv, Quantum Electronics (Wiley, New York, 1967).

[51] K. Blum, Density Matrix Theory and Applications, Vol. 64 (Springer, New York, 2012).

[52] D. Weeraddana, M. Premaratne, S. D. Gunapala, and D. L. Andrews, J. Chem. Phys. 147, 074117 (2017).

[53] S. G. Kosionis, A. F. Terzis, V. Yannopapas, and E. Paspalakis, J. Phys. Chem. C 116, 23663 (2012). 
[54] A. M. Funston, C. Novo, T. J. Davis, and P. Mulvaney, Nano Lett. 9, 1651 (2009).

[55] P. B. Johnson and R.-W. Christy, Phys. Rev. B 6, 4370 (1972).

[56] F. Thiele, C. Fuchs, and R. v. Baltz, Phys. Rev. B 64, 205309 (2001).

[57] S. Schmitt-Rink, D. A. B. Miller, and D. S. Chemla, Phys. Rev. B 35, 8113 (1987).

[58] A. E. Cohen and S. Mukamel, J. Phys. Chem. A 107, 3633 (2003).
[59] L. C. K. Arachchige, Quantum plasmonic analysis of spasers, Ph.D. thesis, Monash University, Clayton, Australia, 2020, https://doi.org/10.26180/5ee9943e64cee.

[60] V. R. S. K. Mudiyanselage, Analysis of scattering properties of nanohybrids with non-spherical nanoresonators and coupled quantum emitters, Ph.D. thesis, Monash University, Clayton, Australia, 2020, https://doi.org/10.26180/5ed9b606c0035.

[61] J. Hong, B.-S. Kim, B. Hou, Y. Cho, S. H. Lee, S. Pak, S. M. Morris, J. I. Sohn, and S. Cha, Plasmonics 15, 1007 (2020). 